\title{
Complete model of a spherical gravitational wave detector with capacitive transducers. Calibration and sensitivity optimization.
}

\author{
Luciano Gottardi \\ LION, Institute of Physics, Kamerlingh Onnes Laboratorium, \\ Leiden University, Leiden, The Netherland**
}

(Dated: June 11, 2018)

\begin{abstract}
We report the results of a detailed numerical analysis of a real resonant spherical gravitational wave antenna operating with six resonant two-mode capacitive transducers read out by superconducting quantum interference devices (SQUID) amplifiers. We derive a set of equations to describe the electro-mechanical dynamics of the detector. The model takes into account the effect of all the noise sources present in each transducer chain: the thermal noise associated with the mechanical resonators, the thermal noise from the superconducting impedance matching transformer, the back-action noise and the additive current noise of the SQUID amplifier. Asymmetries in the detector signal-to-noise ratio and bandwidth, coming from considering the transducers not as point-like objects but as sensor with physically defined geometry and dimension, are also investigated. We calculate the sensitivity for an ultracryogenic, 30 ton, 2 meter in diameter, spherical detector with optimal and non-optimal impedance matching of the electrical read-out scheme to the mechanical modes. The results of the analysis is useful not only to optimize existing smaller mass spherical detector like MiniGrail, in Leiden, but also as a technological guideline for future massive detectors. Furthermore we calculate the antenna patterns when the sphere operates with one, three and six transducers. The sky coverage for two detectors based in The Netherlands and Brasil and operating in coincidence is also estimated. Finally, we describe and numerically verify a calibration and filtering procedure useful for diagnostic and detection purposes in analogy with existing resonant bar detectors.

PACS numbers: 04.80.Nn, 95.55.Ym,07.07Mp,02.60.Pn
\end{abstract}

\footnotetext{
${ }^{*}$ Current address: SRON, National Institute for Space Research, High Energy Astrophysics Division, Utrecht, the Netherlands; Electronic address: l.gottardi@sron.nl
} 


\section{INTRODUCTION}

Resonant bar antennas are in continuous operation at $4.2 K$ with sensitivity and bandwidth never reached before [1],[2]. These detectors could improve their sensitivity of one order of magnitude in the coming years when operating at $100 \mathrm{mK}$. The first ultracryogenic spherical gravitational wave detectors [3, 4] are currently completing their engineering phase and will soon be operational with an expected sensitivity better than $10^{-21} H z^{-1 / 2}$ at $3 k H z$. Much interest is now directed towards the next generation of acoustic detectors, which will be large mass spheres [5] equipped with traditional resonant transducers and broadband dual resonators [6, 7]. The resonant spheres rely on available technology [1, 2], a rather extensive theoretical work [8, 9, 10, 11, 12, 13], and the ground-breaking experimental work performed so far on the ultracryogenic small mass sphere Minigrail [3]. The wide-band dual detector potentially solves the problem of relative narrow bandwidth of current resonant bar and spheres, but the required technology needs to be assessed in separate experiments [7]. Here we numerically analyse the sensitivity and performance of an ultracryogenic spherical detector, equipped with capacitive, SQUID-based, resonant transducers. A general analysis of the problem of the read-out system of for linear detector is given in [14, 15]. Previous works [10, 12, 13, 16, 17, 18] have provided a solution for the read-out and inverse problem of a spherical detector, deriving equations of motion for the five degenerate quadrupole spheroidal modes coupled to $\mathrm{N}$ identical, point-like, single-mode resonant transducers located at arbitrary points on the sphere surface. The effects of transducer asymmetries on the strain sensitivity and bandwidth was studied in [10, 13] for rather

generic radial, point-like, single mode, identical resonators. In [19] the strain sensitivity for a spherical gravitational wave detector with a three-mode inductive transducer with optimal parameters is calculated.

The present paper shows the results of a detailed numerical calculation of the performance of a spherical detector, which uses 2-mode capacitive transducers where the electrical resonant mode of a superconducting matching network can be tuned to the resonant mechanical modes. The signal current from the superconducting matching transformer is read-out by sensitive SQUID amplifiers. We chose this transduction system mainly for two reasons: first, most existing bar and spherical antenna use capacitive transducers, second, the technology involved is so far the most advanced. As recently experimentally demonstrated on the cur- 
rently most sensitive resonant antenna AURIGA [1], such a read-out scheme enhances both the sensitivity and the bandwidth of a resonant detector when working in the tuned mode. Two-stage SQUID amplifiers coupled to a high quality factor load can reach nowadays an energy resolution only an order of magnitude higher than its quantum limit when properly cooled down to $100 \mathrm{mK}$ [20]. Two-stage SQUID amplifiers operates at 4-5K on the bar antenna AURIGA [1], and on the spherical antenna MiniGRAIL [21], with an unprecedently reached sensitivity of about $600 \hbar$.

We use the analysis presented here to study the detector sensitivity as a function of the SQUIDs, the superconducting matching network and the mechanical resonators intrinsic parameters, and to define the optimal coupling between antenna, transducer and amplifiers. The simulations consider the effect of all the parameters involved in a real detector, including the effect of the cold damping network used in the fluxed lock-loop (FLL) of the SQUID amplifier to stabilize the read-out of high Q loads[22]. We study also the sensitivity and signal bandwidth deterioration coming from the transducer being a geometrically extended object rather than a point-like mass. Finally we describe and numerically test a method to fully calibrate a spherical detector and to derived the optimal filter parameters from the experimental data. This method is a generalization of the one used with the resonant bar antenna AURIGA 23]. The codes generated to perform such an analysis can be used as a guideline for the development of future detectors as well as a tool to evaluate the performance of present small mass spherical detectors.

In section \we give an overview of the equations necessary to describe the coupling of a gravitational wave to a sphere and of a sphere to $\mathrm{N}$ mechanical resonator following the formalism introduced by Johnson and Merkowitz [16]. We complete the equation of motion for a capacitive transducer coupled to a SQUID amplifier through a superconducting matching transformer. The detector strain sensitivity, noise temperature and signal bandwidth are derived using the generilized vector approach proposed by Stevenson [13]. This method is particularly powerful when transducers are not identical not only in their mechanical parameters, but also with respect to their noise sources. It indicates a rather simple method to handle the correlation between transducer output channels and to form statistically independent channels (the mode channel concept used in [16]) in absence of symmetries. In section III we describe all the noise sources acting on the sphere and the transducer chain. The results of the numerical analysis are reported in section IV]. First, following the de- 
scription of a parameter optimization procedure, we calculate the strain sensitivity for an ultracryogenic spherical detector, $2 \mathrm{~m}$ in diameter, made of a copper and alluminum alloy ( $C u A l 6 \%)$, and equipped with one and six transducers located in the positions of the Trun-

cated Icosahedral (TI) configuration proposed by Johnson and Merkowitz [16]. Secondly, the antenna patterns are calculated for two spherical detectors with optimal sensitivity located in the north and south hemisphere of the earth. The sky coverage is estimated for such spheres operating with one, three and six resonators, both when working independently and in coincidence. Finally, the effect on the sensitivity is evaluated when non-identical and non-ideal transducers are employed. In section $\nabla$ a complete calibration procedure for a spherical detector is described. First we show a way to derived experimentally the equivalent temperature of the mechanical modes of a sphere equipped with six transducers. Then we discuss a procedure to measure the detector transfer functions and to calibrate its force response. Such a method is finally tested by calculating the detector mode channels response with simulated gravitational wave bursts. Finally, section VI summarizes the results.

\section{SPHERICAL GW RESONANT DETECTORS}

In this section we describe the model used to derive the sensitivity of a spherical gravitational wave antenna with $\mathrm{N}$ transducers coupled only to radial motion. We consider a capacitive transducer, which can be operated in a 2-mode configuration when the electrical mode is tuned to the mechanical one.

The dynamics of a sphere coupled to radial transducers and interacting with gravitational waves is described below using the mode channels formalism introduced by Johnson and Merkowitz [16], and matrices notation suitable to derive the signal-to-noise ratio, SNR, and the noise temperature of a multichannel system [13].

\section{A. Coupling of a bare sphere with the gravitational field}

A gravitational wave is a time-dependent deviation of the metric perturbation. In the coordinate frame of the wave, denoted here by primed coordinates and indices, with the origin at the detector center of mass and the $z^{\prime}$ axis aligned with the propagation direction 
of the wave, the spatial metric perturbation in the TT gauge is given by

$$
\mathbf{H}^{\prime}(t)=\left(\begin{array}{ccc}
h_{+}(t) & h_{\times}(t) & 0 \\
h_{\times}(t) & -h_{+}(t) & 0 \\
0 & 0 & 0
\end{array}\right),
$$

with $h_{+}$and $h_{\times}$corresponding to the two independent wave polarizations. A wave with polarization $h_{+}$deforms a test ring into an ellipse with axes in the $x$ and $y$ directions. A wave with polarization $h_{\times}$deforms the ring at a 45-degree angle to the $x$ and $y$ directions. A circularly polarized wave has $h_{+}= \pm h_{\times}$and rotates the deformation of a test ring in the right-handed (or left-handed) direction [24].

The dynamics of a bare resonant sphere can be described by ordinary elastic theory. A forces $f$ acting on the body will cause a displacement of the sphere mass element at its equilibrium position. The mechanics is described by the standard equations of motion of a forced oscillator. In this section we limit ourselves to review the main result. More complete treatments can be found in [8, 9, 11, 12, 16]. In particular we make use of the formalism introduced by Merkowitz [10, 16].

Denoting by $\mathbf{f}(\mathbf{x}, t)$ the total force that acts on the sphere, including the gravitational wave force, at the position $\mathbf{x}=\{x, y, z\}$ and time $t$, one finds that the equation for each mode amplitude is the one for a forced harmonic oscillator. After Fourier transforming, each mode amplitude can be written as

$$
\begin{gathered}
\mathbf{a}_{\mathbf{m}}(\omega)=\frac{3}{4 \rho \pi R^{3}} \frac{1}{\omega_{m}^{2}-\omega^{2}+j \omega_{m}^{2} \Phi_{m}} \times \\
\quad \times \int \Psi_{l m}(\mathbf{x}) \mathbf{f}(\mathbf{x}, \omega) d^{3} x,
\end{gathered}
$$

where $\mathrm{R}$ is the radius of the sphere and $\rho$ the density. On the right hand side of the equation, the first factor is an arbitrary normalization constant. The second factor describes the oscillating nature of the displacement where $\omega_{m}$ and $\Phi_{m}=1 / Q_{m}$ are, respectively the resonance frequency and the loss angle associated with the quality factor $Q_{m}$ of the $m$ th mode. The integral is calculated over the entire volume of the sphere where $\boldsymbol{\Psi}_{l, m}(\mathbf{x})$ are the time independent orthogonal elastic eigenfunctions of the sphere with $l=0$ or $l=2$.

In general relativity only 5 quadrupolar modes of vibration $(\ell=2)$ will strongly couple to the force density of a gravitational wave due to the fact that the tensor $\mathbf{H}^{\prime}$ is traceless. 
In a perfect sphere they are all degenerate, having the same angular eigenfrequency $\omega_{0}$. The quadrupolar modes can be written in terms of the convenient set of the five real spherical harmonics $Y_{m}(\theta, \phi)$, which are defined as follows:

$$
\left(\begin{array}{l}
Y_{1} \\
Y_{2} \\
Y_{3} \\
Y_{4} \\
Y_{5}
\end{array}\right)=\sqrt{\frac{15}{16 \pi}}\left(\begin{array}{c}
\cos 2 \phi \sin ^{2} \theta \\
\sin 2 \phi \sin ^{2} \theta \\
\sin \phi \sin 2 \theta \\
\cos \phi \sin 2 \theta \\
\frac{1}{\sqrt{3}}\left(3 \cos ^{2} \theta-1\right) .
\end{array}\right)
$$

They are the result of a linear combination of the usual complex-valued spherical harmonics $Y_{2 m}$.

For a sphere of radius $R$ the eigenfunctions can be written as:

$$
\Psi_{m}=[\alpha(r) \hat{\mathbf{r}}+\beta(r) R \nabla] Y_{m}(\theta, \phi)
$$

The radial eigenfunctions $\alpha(r)$ and $\beta(r)$ determine the motion in the radial and tangential directions, respectively. An explicit description of the motion in the radial and tangential directions is given by Ashby and Dreitlein [8] .

In the lab-frame with origin at the center of mass of the detector and the $\mathrm{z}$ axis aligned with the local vertical, a gravitational wave produces an effective time dependent tidal force $\mathbf{F}_{\mathbf{m}}^{\mathbf{S}}$ on each mode $m$ of the sphere equal to the overlap integral of Eqs. (2)). One finds

$$
\begin{aligned}
F_{m}^{S}(t) & =\sqrt{\frac{4 \pi}{15}} \rho \ddot{h}_{m}(t) R^{4}\left[c J_{2}(q R)+3 d J_{2}(k R)\right] \\
& =\frac{1}{2} \ddot{h}_{m}(t) m_{S} \chi R
\end{aligned}
$$

where $J_{2}$ is the spherical Bessel function of order 2, the coefficients $c, d$ specify the shape of the eigenfunctions and are weakly dependent on the material Poisson ratio [9], $m_{S}$ is the physical mass of the sphere and $R \chi$ is the effective length of each mode where $\chi$ depends on the Poisson ratio and is equal to 0.327 for the $\mathrm{CuAl}$ sphere considered in the following analysis. $h_{m}$ are the spherical amplitudes [16], a complete and orthogonal representation of the metric perturbation. $q$ and $k$ are respectively the longitudinal and transverse wave vectors as defined in [8]. The effective force $F_{m}^{S}$ on the corresponding mode of a sphere is therefore uniquely determined by each spherical component of the gravitational field. 
The force acting on each spheroidal mode $m$ in the lab frame can now be written in terms of the gravitational wave amplitudes

$$
\mathbf{F}_{\mathbf{m}}^{\mathbf{S}}=\frac{1}{2} m_{S} \chi R \omega^{2} \mathbf{T}_{\mathbf{V}}\left(\begin{array}{c}
h_{+} \\
h_{\times}
\end{array}\right),
$$

where $\mathbf{T}_{\mathbf{V}}$, given by

$$
\begin{gathered}
\mathbf{T}_{\mathbf{V}}=\left(\begin{array}{cc}
\frac{1}{2}\left(1+\cos ^{2} \theta\right) \cos 2 \phi & \cos \theta \sin 2 \phi \\
-\frac{1}{2}\left(1+\cos ^{2} \theta\right) \sin 2 \phi & \cos \theta \cos 2 \phi \\
-\frac{1}{2} \sin 2 \theta \sin \phi & \sin \theta \cos \phi \\
\frac{1}{2} \sin 2 \theta \cos \phi & \sin \theta \sin \phi \\
\frac{\sqrt{3}}{2} \sin ^{2} \theta & 0
\end{array}\right) \times \\
\times\left(\begin{array}{cc}
\cos 2 \psi & \sin 2 \psi \\
-\sin 2 \psi & \cos 2 \psi
\end{array}\right),
\end{gathered}
$$

is the transformation matrix, which converts the gravitational wave amplitude in the wave frame into spherical amplitude in the lab frame. Here we used the y-convention of the Euler angles shown in figure 1 and the linear combination of the spherical harmonics described in Eqs. (3). The angle $\psi$ is the first Euler angle in the rotation relating the wave frame to the laboratory frame and it carries information about the GW polarization.

\section{B. Sphere with $\mathbf{N}$ resonant transducers}

Resonant transducers are used on resonant detectors, either bars or spheres [1, 3], 25], in order to improve their sensitivity and bandwidth. We consider here the same type of transducers as displacement sensors. They consist of a mechanical resonator with the fundamental mode tuned to the quadrupolar modes of the antenna. At resonance, there is a transfer of momentum between the resonator and the antenna, turning small displacements of a large antenna into large displacements of the small resonator.

Let us consider a set of $N$ resonators attached to the sphere at arbitrary positions $\left(\theta_{j}, \Phi_{j}\right)$. The values of the relative radial displacement of the sphere at the transducers location can be grouped together into pattern vectors for a particular mode. These column vectors, in 
turn, may be put together to form a pattern matrix $B_{m j}$ defined by [26],

$$
B_{m j}=\frac{1}{\alpha} \mathbf{r} \cdot \Psi_{m}\left(\theta_{j}, \phi_{j}\right)
$$

where $\alpha$ is the radial eigenfunction introduced in Eqs. (44). One gets

$$
B_{m j}=Y_{m}\left(\theta_{j}, \phi_{j}\right)
$$

Assuming that each resonator is designed to obey the one dimensional harmonic oscillator law, the coupled equations of motion for the sphere modes, written in matrix form, are

$$
\begin{gathered}
{\left[\begin{array}{cc}
m_{S} \mathbf{I} & \mathbf{0} \\
m_{R} \alpha \mathbf{B}^{T} & m_{R} \mathbf{I}
\end{array}\right]\left[\begin{array}{c}
\ddot{\mathbf{a}}(t) \\
\ddot{\mathbf{q}}(t)
\end{array}\right]+\left[\begin{array}{cc}
k_{S} \mathbf{I} & -k_{R} \alpha \mathbf{B} \\
\mathbf{0} & k_{R} \mathbf{I}
\end{array}\right]\left[\begin{array}{l}
\mathbf{a}(t) \\
\mathbf{q}(t)
\end{array}\right]=} \\
=\left[\begin{array}{cc}
\mathbf{I} & -\alpha \mathbf{B} \\
\mathbf{0} & \mathbf{I}
\end{array}\right]\left[\begin{array}{c}
\mathbf{F}^{\mathbf{S}}(t) \\
\mathbf{F}^{\mathbf{R}}(t)
\end{array}\right],
\end{gathered}
$$

where matrices are denoted by bold fonts and capital letters and vectors by bold fonts and low case letters. The vector a has 5 components and the vector $\mathbf{q}$ has $N$ components. They represent the radial displacement of the sphere and the resonator, respectively. For identical sphere modes and identical transducers, the factors $m, k$, indicating respectively mass and spring constants are identical and can be treated as numbers. In reality, each mode has its own mass, quality factor and spring constant, so they have the form of a diagonal matrix with components $m_{i}^{s}, k_{i}^{s}=m_{i}^{s} \omega_{i}^{2}\left(1+i \Phi_{i}(\omega)\right)$ for the sphere, with $i=1 . .5$ and $m_{j}^{r}$ and $\left.k_{j}^{r}=m_{j}^{r} \omega_{j}^{2}\left(1+i \Phi_{j}(\omega)\right)\right)$ for the resonators, with $j=1 . . N$. Here $\omega_{n}$, with $n=i, j$, is the natural frequency and $\Phi_{n}(\omega)$ is the loss angle of each resonant mode. It represents the frequency dependence of the loss of a mode. For commonly observed dissipations in metals [27], losses do not depends on frequency and $\Phi_{n}(\omega)=1 / Q_{n}$ where $Q_{n}$ is the mode quality factor. In the case of viscous damping, due for example to eddy-current effects, the loss angle is proportional to the frequency and is given by $\Phi_{n}(\omega)=\omega / \omega_{n} Q_{n}$. We consider the first dissipation mechanism to describe the losses in the mechanical modes. $\mathbf{F}^{\mathbf{S}}$ and $\mathbf{F}^{\mathbf{R}}$ are the driving forces, which include the gravitational waves contribution as well as the forces generated by noise sources.

Equation (10) fully describes the mechanical system sphere-resonators when the pattern matrix $B_{m j}$ is known. Here we consider the special transducer configuration proposed by 


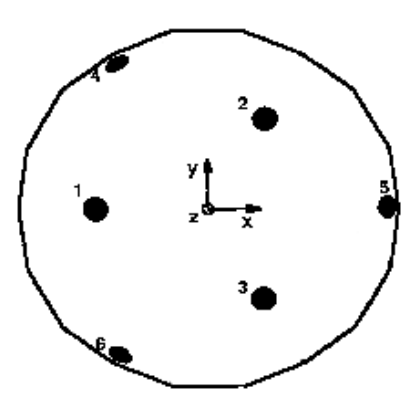

a)

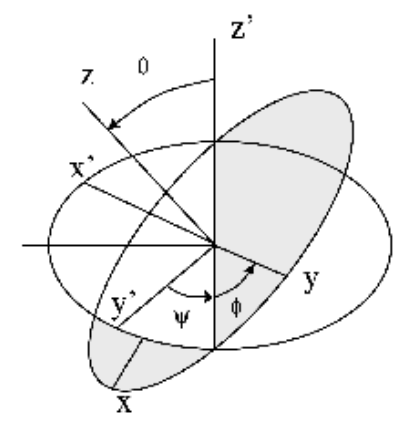

b)

FIG. 1: a) The truncated icosahedral (TI) arrangement for a spherical gravitational wave antenna with resonator locations indicated. The numbering of the resonators corresponds to the ordering used in the numerical calculations. b) Euler angle transforms convention.

Johnson and Merkowitz [16, 28]. It consists of a set of six transducers placed on the 6 pentagonal faces of a Truncated Icosahedron (TI). The resonators are located at two polar angles, $\theta_{T I}=37.3773^{\circ}$ and $79.1876^{\circ}$ as illustrated in figure 1. Their azimuthal angles $\psi_{T I}$ are multiples of $60^{\circ}$.

Below we derive the complete equations of motion for a spherical detector equipped with capacitive transducer and SQUID amplifiers. In a capacitive transducer, the resonating mass, tuned to the spheroidal modes, modulates the charge of a parallel plate capacitor biased at a large constant voltage. The capacitor is formed by the resonating mass top surface and an electrode, assembled with a gap of the order of tens of micrometers. The input coil of the dc-SQUID chip is coupled to the capacitive transducer via a high- $Q$ superconductive transformer, which can have, eventually, the electric resonance coupled to the mechanical modes in order to enhance the bandwidth. The superconducting transformer is essential to match the low impedance of the SQUID with the high impedance of the capacitor. In the model, which is schematically shown in figure 2, we include the relevant Gaussian noise sources of the read-out scheme.

\section{Equation of motion of a spherical detector with resonant capacitive transducers}

The motion equations of a single capacitive transducer coupled to one mechanical mode of the sphere can be generalized to include the complete mechanical response of a sphere coupled to $N$ resonators, described by Eqs. (10), and all the equations for the electrical 


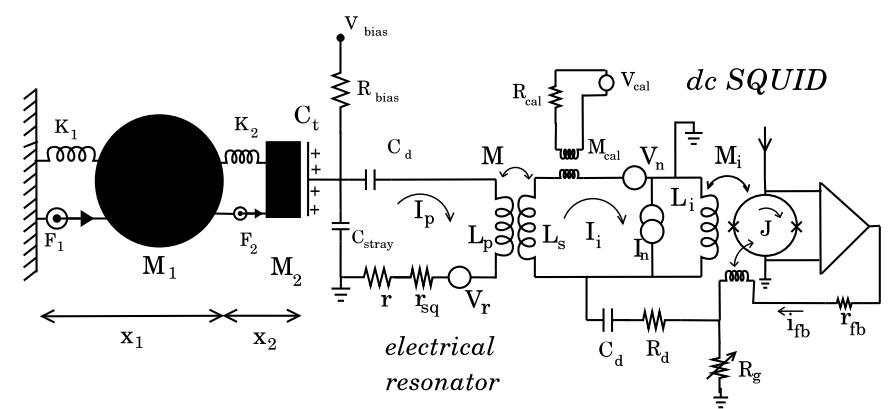

FIG. 2: Single mode electro-mechanical model of a spherical antenna with mechanical resonator and capacitive transducer coupled to a SQUID through a superconducting matching transformer.

circuit of each resonator. After Fourier transforming, Eqs. (10) can be simplified defining the following $5+N$ square matrices:

$$
\begin{aligned}
& \mathbf{M}=\left[\begin{array}{cc}
m_{S} \mathbf{I} & \mathbf{0} \\
m_{R} \alpha \mathbf{B}^{T} & m_{R} \mathbf{I}
\end{array}\right], \quad \mathbf{K}=\left[\begin{array}{cc}
k_{S} \mathbf{I} & -k_{R} \alpha \mathbf{B} \\
\mathbf{0} & k_{R} \mathbf{I}
\end{array}\right], \\
& \mathbf{A}=\left[\begin{array}{cc}
\mathbf{I} & -\alpha \mathbf{B} \\
\mathbf{0} & \mathbf{I}
\end{array}\right] .
\end{aligned}
$$

They are respectively the mass, elastic and force matrices of the coupled sphere. The force matrix A describes the mechanical coupling between the $5+\mathrm{N}$ resonant modes of the detector. We can finally write

$$
\left[-\omega^{2} \mathbf{M}+\mathbf{K}\right]\left[\begin{array}{c}
\mathbf{a}(\omega) \\
\mathbf{q}(\omega)
\end{array}\right]=\mathbf{A}\left[\begin{array}{c}
\mathbf{F}_{\mathbf{N}}^{\mathbf{S}}(\omega) \\
\mathbf{F}_{\mathbf{N}}^{\mathbf{R}}(\omega)
\end{array}\right] .
$$

We assume that each of the $N$ transducers mounted on the sphere has the same electrical circuit configuration described in figure 2, but not necessarily the same value of the parameters. We shall define then the vectors $\mathbf{I}_{\mathbf{p}}(\omega)=\left(I_{p, 1} . . I_{p, N}\right), \mathbf{I}_{\mathbf{i}}(\omega)=\left(I_{i, 1} . . I_{i, N}\right), \mathbf{V}_{\mathbf{r}}(\omega)=\left(V_{r, 1} . . V_{r, N}\right)$, $\mathbf{V}_{\mathbf{n}}(\omega)=\left(V_{r, 1} . . V_{n, N}\right)$, which describe, for each transducer, respectively the current in the superconducting matching transformer, the current in the input coil of the dc SQUID amplifier, the voltage noise generated in the $L C$ superconducting resonators and the voltage noise of the dc SQUID amplifiers.

Denoting by $E_{i}$ the electric field stored in the $i$ th capacitive transducer, we define the electric field matrix as follows:

$$
\mathbf{E}=\operatorname{diag}\left(E_{1} . . E_{N}\right)
$$


The electrical circuit equations can be written in matrix form as follows

$$
\left[\begin{array}{ccc}
\mathbf{E} & \mathbf{Z}_{11} & \mathbf{Z}_{12} \\
\mathbf{0} & \mathbf{Z}_{21} & \mathbf{Z}_{22}
\end{array}\right]\left[\begin{array}{c}
\mathbf{q}(\omega) \\
\mathbf{I}_{\mathbf{p}}(\omega) \\
\mathbf{I}_{\mathbf{i}}(\omega)
\end{array}\right]=\left[\begin{array}{ll}
\mathbf{I} & 0 \\
\mathbf{0} & \mathbf{I}
\end{array}\right]\left[\begin{array}{c}
\mathbf{V}_{\mathbf{r}}(\omega) \\
\mathbf{V}_{\mathbf{n}}(\omega)
\end{array}\right]
$$

We chose the electric matrix $\mathbf{E}$ to be diagonal, because we made the reasonable assumption that the electric field force acts only on the resonator which the field is applied to. The impedance matrix

$$
\mathrm{Z}=\left[\begin{array}{ll}
\mathrm{Z}_{11} & \mathrm{Z}_{12} \\
\mathrm{Z}_{21} & \mathrm{Z}_{22}
\end{array}\right]
$$

is a $(N+N) \times(N+N)$ matrix. Each of the four $N \times N$ matrices $\mathbf{Z}_{\mathbf{i j}}$ is diagonal if we consider as negligible the possible crosstalk between the read-out electronics of each transducer. Each diagonal member of $\mathbf{Z}_{\mathbf{i j}}$ is equal to

$$
\begin{aligned}
& Z_{11}^{i}=r^{i}+r_{s}^{i}+j \omega L_{p}^{i}+\frac{1}{j \omega C_{p}^{i}}, \quad Z_{12}^{i}=-j \omega M^{i} \\
& Z_{21}^{i}=-j \omega M^{i}, \quad Z_{22}^{i}=j \omega\left(L_{s}^{i}+L_{i n}^{i}\right),
\end{aligned}
$$

where $r^{i}$ is a resistance associated with the losses in the superconducting resonator. $C_{p}^{i}$ is the total transducer capacitance resulting from the parallel between the transducer and parasitic capacitance. $r_{s}$ is a lossless resistance resulting from operating the SQUID amplifier in flux locked loop (FLL). There is no thermal noise contribution associated to $r_{s}$ because it is the result of a feedback mechanism. Such a resistance can be controlled by implementing a cold-damping system [22]. In this way the detector has a virtual low quality factor making the FLL electrically stable. We remark that introducing such a damping scheme in our calculations brought benefit to the numerical analysis, by eliminating the computational problem of sharp resonances.

To fully describe the mechanical and electrical dynamics of the detector we have to introduce the back-action of the electrical read-out circuit on the mechanical system. The current flowing in the $L C$ loop of the circuit in figure 2 generates a force on the mechanical resonator proportional to the current itself and the applied electric field. The back-action force vector $\mathbf{F}_{\mathbf{B} \mathbf{A}}^{\mathbf{R}}(\omega)$ adds to the Langevin force generators $\mathbf{F}_{\mathbf{N}}^{\mathbf{R}}(\omega)$ introduced in Eqs. (12) and is equal to

$$
\mathbf{F}_{\mathbf{B A}}^{\mathbf{R}}(\omega)=\left(\frac{E_{1} I_{p, 1}}{j \omega} . . \frac{E_{N} I_{p, N}}{j \omega}\right)=\frac{\mathbf{E I}_{\mathbf{p}}(\omega)}{j \omega} .
$$


The complete set of coupled equations of motion becomes finally:

$$
\left[\begin{array}{cccc}
\mathcal{M} & \mathbf{Z}_{\mathbf{B A}} & \mathbf{0} \\
\mathbf{0} & \mathbf{E} & \mathbf{Z} \\
\mathbf{0} & \mathbf{0} & &
\end{array}\right]\left[\begin{array}{c}
\mathbf{a}(\omega) \\
\mathbf{q}(\omega) \\
\mathbf{I}_{\mathbf{p}}(\omega) \\
\mathbf{I}_{\mathbf{i}}(\omega)
\end{array}\right]=\mathbf{A}^{\prime}\left[\begin{array}{c}
\mathbf{F}_{\mathbf{N}}^{\mathbf{S}}(\omega) \\
\mathbf{F}_{\mathbf{N}}^{\mathbf{R}}(\omega) \\
\mathbf{V}_{\mathbf{r}}(\omega) \\
\mathbf{V}_{\mathbf{n}}(\omega)
\end{array}\right]
$$

where $\mathcal{M}=-\omega^{2} \mathbf{M}+\mathbf{K}, \mathbf{Z}_{\mathbf{B A}}$ is the $(5+N) \times N$ back-action matrix given by

$$
\mathbf{Z}_{\mathbf{B A}}=\left[\begin{array}{c}
-\alpha \mathbf{B} \\
\mathbf{I}
\end{array}\right] \frac{\mathbf{E}}{j \omega}
$$

and

$$
\mathbf{A}^{\prime}=\left[\begin{array}{ll}
\mathbf{A} & 0 \\
0 & \mathbf{I}
\end{array}\right]
$$

The $(5+3 N)$ square matrix on the left side of Eqs. (18) can be seen as the impedance matrix $\mathcal{Z}$ of the electro-mechanical system. Defining $\mathbf{G}=\mathcal{Z}^{-1} \mathbf{A}^{\prime}$, the SQUID input current for each transducer is given by

$$
\mathbf{I}_{\mathbf{i}}=\mathbf{G}_{\mathbf{I}}\left[\begin{array}{c}
\mathbf{F}_{\mathbf{N}}^{\mathbf{S}}(\omega) \\
\mathbf{F}_{\mathbf{N}}^{\mathbf{R}}(\omega) \\
\mathbf{V}_{\mathbf{r}}(\omega) \\
\mathbf{V}_{\mathbf{n}}(\omega)
\end{array}\right]=\mathbf{G}_{\mathbf{I}} \mathbf{F}
$$

where $\mathbf{G}_{\mathbf{I}}$ is a submatrix of the admittance matrix $\mathbf{G}$ with components $G_{s, r}$, where $s=$ $5+2 N . .5+3 N$ and $r=1 . .5+3 N$. The vectors $\mathbf{F}^{\mathbf{S}}, \mathbf{F}^{\mathbf{R}}, \mathbf{V}_{\mathbf{r}}$ and $\mathbf{V}_{\mathbf{n}}$ are the forces generated by each noise source.

The noise of the detector, referred to the SQUID amplifier input, in absence of signal, is described by the spectral density matrix $\mathbf{S}_{\mathbf{I}}[29]$. Each component of the matrix,

$$
S_{I}^{m n}=\int_{-\infty}^{\infty} e^{-j \omega \tau} R_{n m}(\tau) d \tau=\left\langle I_{I}^{m}(\omega) I_{I}^{n *}(\omega)\right\rangle
$$

is the Fourier transform of the correlation function for the $m$ th and $n$th outputs defined as

$$
R_{n m}(\tau)=\left\langle I_{I}^{m}(t) I_{I}^{n}(t-\tau)\right\rangle=\int_{-\infty}^{\infty} I_{I}^{m}(t) I_{I}^{n}(t-\tau) d t .
$$

From Eqs. (23), each component of the spectral density matrix becomes the product of the Fourier transforms of the transducer outputs, as shown in the second equality of Eqs. (22). 
The white current noise $I_{0}^{n}$ of the SQUID amplifier, which will be better defined below, needs to be added to the spectral matrix $S_{I}$. In matrix notation we can easily write the total SQUID current spectral density matrix as

$$
\mathrm{S}_{\mathrm{I}}=\mathrm{G}_{\mathbf{I}} \mathrm{FF}^{*} \mathrm{G}_{\mathrm{I}}^{*}+\mathrm{S}_{\mathbf{I}, \mathbf{0}}
$$

where the $\mathrm{N}$ square matrix $\mathbf{S}_{\mathbf{I}, \mathbf{0}}$ has components $S_{I, 0}^{m, n}=I_{0}^{m} I_{0}^{n *}$. The diagonal elements of $S_{I, 0}$ are equal to the current spectral density given in Eqs. (36). The correlation between the $N$ SQUIDs additive current noise is expected to be negligible so, in the following, we will consider diagonal the matrix $\mathbf{S}_{\mathbf{I}, \mathbf{0}}$. The spectral density defined in Eqs. (24) can be numerically calculated and experimentally measured by means of techniques where the phase information is preserved.

The optimal signal to noise ratio $\rho_{0}$, for a gravitational wave signal of amplitude $\tilde{h}(\omega)$, is given by

$$
\rho_{0}^{2}=4 \int_{0}^{\infty} \frac{\tilde{h}^{2}(\omega)}{S_{h h}(\omega)} \frac{d \omega}{2 \pi}
$$

where

$$
\begin{aligned}
S_{h h}(\omega)=\mathbf{h}^{*}(\omega)\left[\left(\mathbf{F}_{\mathbf{m}}^{\mathbf{S}}\right)^{*} \mathbf{G}_{\text {sig, }, \mathbf{I}}^{*} \mathbf{S}_{\mathbf{I}}^{-\mathbf{1}}(\omega) \mathbf{G}_{\mathbf{s i g}, \mathbf{I}} \mathbf{F}_{\mathbf{m}}^{\mathbf{S}}\right]^{-1} \mathbf{h}(\omega) & \\
= & \frac{4}{\left(m_{S} \chi R \omega^{2}\right)^{2}}\left[\mathbf{T}_{\mathbf{V}}^{*} \mathbf{G}_{\mathbf{s i g}, \mathbf{I}}^{*} \mathbf{S}_{\mathbf{I}}^{-\mathbf{1}}(\omega) \mathbf{G}_{\mathbf{s i g}, \mathbf{I}} \mathbf{T}_{\mathbf{V}}\right]^{-1}
\end{aligned}
$$

is the one-sided total strain noise power spectrum.

In the equation above, we called $\mathbf{h}$ the vector $\left(h_{+} h_{\times}\right)$and the $N \times 5$ matrix $\mathbf{G}_{\mathbf{s i g}, \mathbf{I}}$ is a submatrix of the admittance matrix $\mathbf{G}(\omega)$ with components $G_{s, r}$, where $s=5+2 N+1 . .5+3 N$ and $r=1 . .5$.

Each transducer line should be considered as a linear system with the $(5+2 N+2)$ uncorrelated noise sources described above, if we consider as negligible the correlation between the voltage and current noise in the SQUID amplifier. However, the outputs of the N transducers do have correlated noise and the off-diagonal components of the spectral density matrix are non zero. One can always find linear combinations of transducers outputs, which produce $\mathrm{N}$ uncorrelated signals [13]. Since $S_{I}$ is an $N \times N$ Hermitian matrix it can be diagonalized by an unitary matrix $U(\omega)$. A new output channel vector is then obtained and is related to the original vector $\mathbf{I}_{\mathbf{i}}$ by

$$
\mathbf{I}_{\mathbf{i}}^{\mathbf{u}}=\mathbf{U}^{*} \mathbf{I}_{\mathbf{i}}
$$


The channels $\mathbf{I}_{\mathbf{i}}^{\mathbf{u}}$ are statistically independent and the spectral density matrix

$$
\mathbf{S}_{\mathbf{i}}^{\mathbf{u}}=\mathbf{U}^{*} \mathbf{S}_{\mathbf{i}} \mathbf{U}=\operatorname{diag}\left(\psi_{1}(\omega), \ldots, \psi_{N}(\omega)\right)
$$

is diagonal, the eigenvalues $\psi_{i}$ of $\mathbf{S}_{\mathbf{i}}^{\mathbf{u}}$ being the noise spectral density of each independent channel.

We notice that, after performing the diagonalization in Eqs. (28), the total optimal SNR can be written as the sum of the SNR in each statistically independent output channel.

$$
\rho_{0}^{2}=\sum_{i=1}^{N} 4 \int_{0}^{+\infty} \frac{\left|I_{i}^{u, s i g}\right|^{2}}{\psi_{i}} \frac{d \omega}{2 \pi} .
$$

From Eqs. (25) and Eqs. (26) the SNR becomes

$$
\begin{aligned}
\rho_{0}^{2}=\left(\frac{m_{s} \chi R \omega_{0}^{2}}{2}\right)^{2} \frac{|\tilde{h}(\omega)|^{2}}{2 \pi} \times & \\
& \times\left[4 \int_{0}^{+\infty} \mathbf{T}_{\mathbf{V}}^{*} \mathbf{G}_{\mathbf{s i g}, \mathbf{I}}^{*}(\omega) \mathbf{S}_{\mathbf{I}}^{-1}(\omega) \mathbf{G}_{\mathbf{s i g}, \mathbf{I}}(\omega) \mathbf{T}_{\mathbf{V}} d \omega\right] .
\end{aligned}
$$

The performance of resonant detectors is often characterized by their sensitivity to impulsive burst signals which vary little over the detection bandwidth. For impulsive signals, the SNR is proportional to the deposited energy $E$ in the antenna initially at rest given by, [24],

$$
E=\frac{c^{3}}{G} \frac{1}{16 \pi} \omega_{0}^{2}\left|\tilde{h}\left(\omega_{0}\right)\right|^{2} \Sigma=\frac{\pi}{4} \frac{\rho_{S} v_{S}^{5}}{f_{0}} \Pi|\tilde{h}(\omega)|^{2},
$$

where $\Sigma=\frac{G}{c^{3}} \frac{\rho v_{S}^{5}}{f_{0}^{3}} \Pi$ is the integrated cross section of a spherical detector [9] and $\Pi$ is the reduced energy cross section equal to 0.215 for a $\mathrm{CuAl}$ sphere [12]. $G$ and $c$ are Newton's gravitational constant and the speed of light respectively, while $\rho_{S}$ and $v_{S}$ are the antenna material density and sound velocity.

The pulse detection noise temperature $T_{N}$ is then defined as

$$
T_{N}=\frac{1}{k_{B}} \frac{E}{\rho_{0}^{2}} .
$$

This is a convenient quantity to compare spherical detectors with bar detectors: while $E$ for a bar-antenna depends on source direction and polarization, $T_{N}$ does not. Using Eqs. (30) and Eqs. (31) we can write $T_{N}$ as follows

$$
\begin{aligned}
T_{N}=\frac{4 \pi}{k_{B}}\left(\frac{\pi}{m_{S} \chi R}\right)^{2} \frac{\rho_{S} v_{s}^{5}}{\omega_{0}^{5}} \times & \\
& \times\left[4 \int_{0}^{+\infty} \mathbf{T}_{\mathbf{V}}^{*} \mathbf{G}_{\mathbf{s i g}, \mathbf{I}}^{*}(\omega) \mathbf{S}_{\mathbf{I}}^{-1}(\omega) \mathbf{G}_{\mathbf{s i g}, \mathbf{I}}(\omega) \mathbf{T}_{\mathbf{V}} d \omega\right]^{-1}
\end{aligned}
$$


We remark here that, due to the dependency of the matrix $\mathbf{T}_{\mathbf{V}}$ from the wave direction and polarization, the sensitivity of a spherical detector will be isotropic over the sky only if a sufficient number of transducer is used $(N>5)$. For $N<5$, one can define the detector sensitivity by averaging over the direction and polarization as described in [30].

\section{NOISE CONTRIBUTIONS}

\section{A. Mechanical resonators}

Thermal noise is the main contribution of the mechanical resonators to the total detector noise. The spectral density of the thermally activated forces acting on the mechanical modes can be estimated from the fluctuation-dissipation theorem [31], and described as follows

$$
\mathbf{S}_{\mathbf{F}, \mathbf{t h}}(\omega)=4 k_{B} T R e\left(\frac{\mathcal{M}}{j \omega},\right),
$$

where $\frac{\mathcal{M}}{j \omega}$ is the system mechanical impedance matrix derived in Eqs. (18), $k_{B}$ is the Boltzmann constant and $\mathrm{T}$ is the thermodynamic temperature. With this formalism we take into account the stochastic crosscoupling among the various degrees of freedom of a macroscopic mechanical body [32].

The thermal noise contribution of other modes of the spherical detector has never been considered as a possible source of noise because they lay generally far a way from the detector bandwidth. In a real detector, the first toroidal modes are only a few tens of $\mathrm{Hz}$ lower than the spheroidal modes due to the spread of the resonances caused by the detector asymmetry [33]. Moreover, in a spherical detector with large bandwidth, the toroidal modes will unrecoverably fall into the sensitive bandwidth. However they are not sensitive to GW and they couple very weakly to a resonator with radial sensitivity. The toroidal modes usually have large quality factors than the spheroidal modes and their thermal noise contribution is generally negligible.

Further-more, higher frequency modes could also contribute to the total noise in the detector bandwidth due to down-conversions phenomena related to the physical geometry and dimension of the read-out transducer [34]. To evaluate the contribution of higher frequency

mode one can proceed as in [35], and calculate the total mechanical impedance using Finite Element or numerical techniques. Here we consider only the thermal noise of the spheroidal 
modes and the main radial resonance of the transducers, the latter being usually the dominant source of noise. The contribution from other modes will be studied in a following paper.

\section{B. Electrical resonators}

The LC resonator which derives from transducer capacitance and the primary coil of the matching transformer contributes to the total noise with a thermal voltage noise source associated with the resonator losses, with single-sided spectral density $S_{V}=4 k_{B} T R e\left(Z_{L C}(\omega)\right)$. The dissipating term $r=\operatorname{Re}\left(Z_{L C}(\omega)\right)$ is linked to the intrinsic electrical quality factor of the $\mathrm{LC}$ resonator by the well-known relation $Q_{e l}=\omega_{\text {res }} L_{p} / R e\left(Z_{L C}\right)$. The dissipation resistance $r$ includes the contributions from dielectric losses in the transducer and decoupling capacitor, in the coil parasitic capacitance and in the coil insulating layers and holder [36], magnetic losses due to flux motion in the superconducting shields, "magneto-resistive" losses due to dissipative components in the SQUID amplifier [37].

The thermal noise contribution from the LC resonator adds to the SQUID back-action noise and may become significant when SQUID amplifiers with $\epsilon<200 \hbar$ are employed.

\section{SQUID amplifier noise theory}

A coupled dc SQUID amplifier can be modelled as an ideal current amplifier with a current noise source $I_{n}$ in parallel and a voltage noise source $V_{n}$ in series with the input coil. The two of them are responsible respectively for additive and back action noise. To fully characterise the SQUID it is necessary to estimate both the noise contributions. An useful parameter to characterize an amplifier is its noise temperature $T_{n}$, defined by

$$
T_{n}=\frac{\sqrt{S_{v v} S i i-\operatorname{Im}\left(S_{i v}\right)^{2}}}{4 k_{B}}=\frac{\omega}{k_{B}} \sqrt{\epsilon_{i i} \epsilon_{v v}-\epsilon_{i v}^{2}}
$$

in the classical limit when $k_{B} T_{N}>\hbar \omega . T_{n}$ is the temperature at which the optimal input impedance gives the thermal noise power equal to the amplifier noise. Its minimum value for a linear amplifier is imposed by the uncertainty principle and is given by $T_{N} \operatorname{sim} \hbar \omega / k_{B}$ [38]. $S_{v v}$ and $S_{i i}$ are the spectral densities, referred to the SQUID input coil, of the two noise generators, while $S_{v i}$ is the cross correlation between the two. According to Clarke- 
Tesche-Giffard (CTG) theory the single-sided spectra are equal to

$$
\begin{aligned}
& S_{i i} \simeq 16 \frac{k_{B} T}{R_{s}}\left(\frac{L_{S Q}}{M_{i, S Q}}\right)^{2} \\
& S_{v v} \simeq 11 \frac{k_{B} T}{R_{s}} \omega\left(M_{i, S Q}\right)^{2} \\
& S_{j v} \simeq 12 \frac{k_{B} T}{R_{s}} j \omega L_{S Q},
\end{aligned}
$$

where $R_{s}, L_{S Q}, M_{i, S Q}$ are respectively shunt resistors, self inductance and input coil mutual inductance of the SQUID. The cross correlation power spectrum is always purely imaginary due to the time-reversal simmetry of the SQUID motion equation. Its absolute value is usually small and we will neglect it in the following calculations. In Eqs. (35) the noise temperature is also defined in terms the energy resolutions $\epsilon_{i i}, \epsilon_{v v}$ and $\epsilon_{i v}$ expressed in units of $\hbar$. In the following sections we will not make distinctions between the voltage and current energy resolution, $\epsilon_{i i}$ and $\epsilon_{v v}$, and we will generically talk about SQUID energy resolution assuming that they both have the same value. The cross correlation energy resolution $\epsilon_{i v}$ will be neglected.

\section{NUMERICAL ANALYSIS}

Here we numerically calculate the noise temperature, the bandwidth and the strain sensitivity of an ultracryogenic, large $2 m$ in diameter, spherical detector as a function of the transduction chain parameters. We consider a detector operating according to the state-ofart resonant antenna technology and when all the parameters are improved to operate the detector nearly at the quantum limit. Further we calculate the antenna patterns and the sky coverage of two identical detectors operated in coincidence and located respectively in Leiden (The Netherlands) and Saõ Paolo (Brasil), the location of the two small spherical antenna Minigrail [3], and Mario Schenberg [4], currently under development. Finally the anisotropy in the sensitivity and bandwidth is studied for a not ideal resonator and a not optimally tuned 2-mode capacitive transducer. 


\section{A. Parameters optimization}

To optimize a resonant detector one must know the voltage and current noise of the available SQUID amplifier. The other parameters can then be adjusted to achieve the best SNR. The optimal impedance matching between the mechanical resonators and the SQUID amplifier is achieved when the transducer electrical mode is tuned to the mechanical resonances. In the scheme described in figure 2, the electrical mode contributes to the transducer chain with an equivalent mass $m_{e q}=C_{T} E^{2} / \omega_{e l}^{2}$. In a multimode detector the energy transfer between each resonator is optimized when $\mu=m_{R} / m_{e f f}=m_{e l} / m_{R}$, where $\mu$ is defined as the mass ratio and $m_{e f f}$ is the effective mass ratio of the five spheroidal modes, which is a fraction of the sphere total mass $m_{s}$ and is equal to $m_{e f f}=5 / 6 \chi m_{s}$ [16]. For optimal mass-ratio one gets a shorter energy transfer time between the modes and a wider system bandwidth. To fully describe the sensitivity of a resonant detector we consider the signal-to-noise ratio SNR, the pulse detection noise temperature $T_{N}$ and the signal bandwidth, here described by [39]:

$$
\delta f=\frac{1}{2 \pi} \frac{\left(\int_{0}^{\infty}\left|S_{h h}^{-2}(\omega)\right| d \omega\right)^{2}}{\int_{0}^{\infty}\left|S_{h h}^{-2}(\omega)\right|^{2} d \omega} .
$$

In the analysis below we imposed the following conditions:

$$
\begin{aligned}
& E=\mu \omega_{R}\left(\frac{m_{e f f}}{C_{T}}\right)^{1 / 2}, \\
& \omega_{e l}^{2}=\frac{1}{C_{T} L_{r}}=\omega_{t r}^{2},
\end{aligned}
$$

where $L_{r}=L_{p}\left(1-k^{2} L_{s} /\left(L_{s}+L_{i}\right)\right)$ is the reduced inductance of the primary coil of the matching transformer with coupling $k^{2}=M^{2} /\left(L_{s} L_{p}\right)$. The first condition is obtained by assuming a constant mass ratio $\mu=m_{e l} / m_{R}$. With the second condition we impose that the electrical resonances are tuned to the transducers mechanical resonances. This condition is fundamental to get optimal matching in a capacitive transducer. The parameters of the electrical matching network depend on the value of the SQUID input inductance and noise, and on the detector mass ratio. It can be shown that for large mass ratio, a coupling $k \sim 1$, and optimal matching, the inductance $L_{s}$ of the superconducting transformer secondary coil should be as large as the SQUID input inductance $L_{i}$. However in practice large $L_{s}$ will guarantee large coupling and finally a better sensitivity. For low mass ratio, 
the impedance seen by the transformer primary coil is lower than for high mass ratio when the same capacitance is considered. One can obtain a better matching by decreasing both the inductances $L_{p}$ and $L_{s}$ of the primary and secondary coil while maintaining valid the conditions Eqs. (38). The improvement is however rather limited giving for example about $20 \%$ better noise temperature using an inductance $L_{s} \sim 0.3 L_{i}$ for a mass ratio $\mu=0.001$ and a $k \sim 1$.

We anticipate that for a detector with significant thermal noise (high $T / Q$ ratio) operating with a low noise SQUID amplifier, the optimal sensitivity is obtained for large mass ratio $\mu$ and, consequently, high transducer capacitance (see Eqs. (38)). It is however physically impossible to reach arbitrary resonator high mass and capacitance using the detector configuration discussed here. The maximum transducer capacitance $C_{T}$ and mass $m_{R}$ one can reasonably obtain, which however have never been developed so far, are estimated to be $C_{T, \max } \simeq 30 \mathrm{nF}$ and $m_{R, \max } \simeq 90 \mathrm{Kg}$. They correspond to a transducer with an area $A \simeq 0.1 m^{2}$, a gap $d \simeq 20 \mu m$ and a mass ratio $\mu \simeq 0.01[46]$. We notice that strong physical constrains apply in general also to the mechanical resonant frequency due to the limits on the resonator membrane thickness [40, 41]. However, a resonator "rosette" design as developed for the NAUTILUS and EXPLORER detectors [41], allows a rather wide freedom in the choice of the resonator mass and sensitive area for a give resonant frequency.

We report below the calculated detector sensitivity for a $2 m$ large in diameter, 30 ton, $\mathrm{CuAl}$ sphere. The main parameters of the detector are summarized in table I. Such an antenna has a cross section $\Sigma=9.76 \cdot 10^{-24} m^{2} H z$. The total energy deposited by a GW of amplitude $\tilde{h}(\omega)$ is $E=1.0 \cdot 10^{35} \omega^{2}|\tilde{h}(\omega)|^{2} K$. A GW burst signal, lasting for a time $\tau_{G} 1 m s$ shorter than the detector integration time and rising quickly to an amplitude $h_{0}=$ $\tilde{h}\left(\omega_{0}\right) / \tau_{G} \sim 2 \cdot 10^{-21}$, deposits an energy $T_{G B}=1 \mu K$.

We consider a sphere equipped with six radial capacitive resonators in the TI configuration. The electrical mode of the superconducting matching network is tuned to the mechanical ones. The signal of each transducer is amplified by a two-stage SQUID amplifier. Two situations have been considered. In the first one the detector parameters have values according to the current available technology. In the second we estimate the ultimate sensitivity of the detector obtainable with quantum limited amplifiers and ultra high Q mechanical and electrical resonators. For a given $T / Q$ ratio and SQUID amplifier energy resolution, the detector sensitivity depends on the resonators mass ratio, the electric field 


\begin{tabular}{ccc}
\hline & current & quantum \\
parameters & technology & limit \\
\hline sphere mass, $M_{s}[$ tons $]$ & 30 & 30 \\
sphere diameter $[m]$ & 2 & 2 \\
resonator mass, $M_{r}[K g]$ & 10 & 90 \\
spheroidal modes, $f_{l}[H z]$ & $987,1001,1008,987,1001,1008$, \\
$Q_{s}, Q_{r}, Q_{e l}$ & 1012,1017 & 1012,1017 \\
$T[m K]$ & $2 \cdot 10^{6}$ & $5 \cdot 10^{7}$ \\
$C_{t}[n F]$ & 50 & 20 \\
$L_{p}[H]$ & 10 & 30 \\
bias field, $E[$ Volt $/ m]$ & $5 \cdot 10^{6}$ & $4 \cdot 10^{7}$ \\
SQUID sensitivity, $E_{r e s}[\hbar]$ & 50 & 1 \\
\hline
\end{tabular}

TABLE I: Main parameters used in the numerical analysis of the sensitivity of a spherical detector equipped with six capacitive transducers. The first column shows the parameters of a $2 \mathrm{~m}$ sphere in $\mathrm{CuAl}$ already achieved in separate experiments. In the second column we give the parameters necessary to operate the detector at the quantum limit. The CuAl alloys have a sound velocity of $4700 \mathrm{~m} / \mathrm{s}$ and a Poisson ration of about 0.3 . The material dependent factor $\chi$ introduced in Eqs. (5) is $\chi=0.327$ for $\mathrm{CuAl}$. The effective mass of the spheroidal modes is then $m_{e f f}=$ $5 / 6 \chi * m_{s} \simeq 8$ tons.

bias $E$, the transducers capacitance and the parameters of the matching transformers.

The detector effective noise temperature, the bandwidth and the SNR for a GW burst of $T_{G B}=10 \mu \mathrm{K}$ are calculated as a function of the mass ratio for different values of the $T / Q$ ratio. $T / Q$ refers both to the mechanical and electrical resonances. The equivalent mass of the electrical resonator is adjusted according to the mass ratio by changing the electric field bias E. The optimal electrical field is calculated as a function of the mass ratio and the transducer capacitance. The results are shown in figure 3, We considered mechanical and electrical $T / Q$ of $1 \cdot 10^{-7}, 2.5 \cdot 10^{-8}, 4 \cdot 10^{-10}$. Mechanical $T / Q$ of the order of $10^{-8}$ have been reached in a $\mathrm{CuAl}$ sphere cooled at $50 \mathrm{mK}$ [42], and in Al5056 bars cooled at $100 \mathrm{mK}$. The 


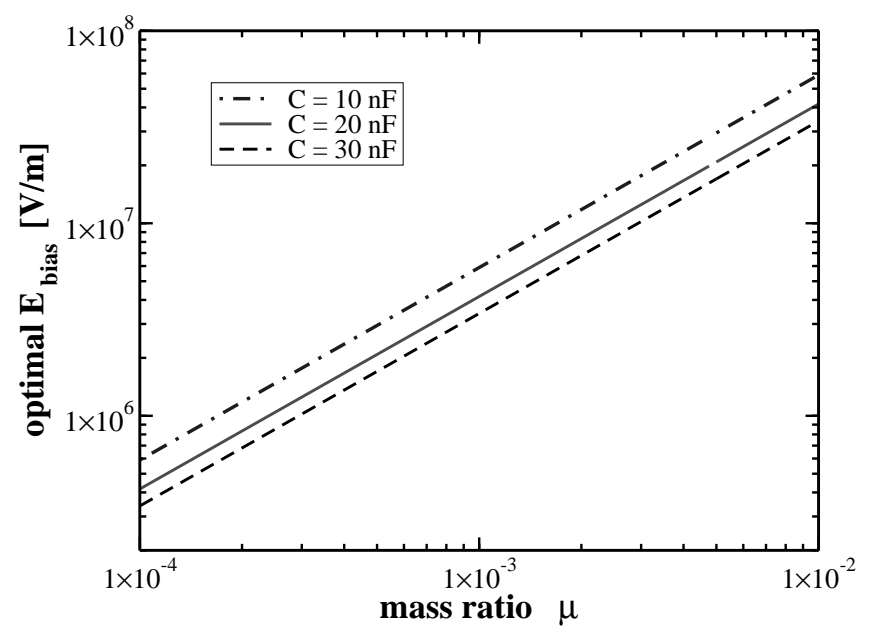

FIG. 3: Optimal bias electric field for different value of the transducer capacitance as a function of the resonators mass ratio $\mu$.

lowest $T / Q=3 \cdot 10^{-8}$ for electrical resonators has been achieved by the AURIGA group with a large $\mathrm{Nb}$ coil resonator cooled down to $50 \mathrm{mK}$ [20]. The fabrication of electrical resonators with $T / Q=1 \cdot 10^{-7}$ at acoustic frequencies is nowadays a well established technology. A $T / Q<10^{-8}$ has not yet been achieved experimentally either in mechanical transducers or in electrical resonators. The results of the calculations are shown in plots figure $4 \mathbf{a}$ ), where the noise temperature, the SNR and the bandwidth are given as functions of the mass and the $T / Q$ ratio. The SQUID amplifier coupled energy resolution was chosen to be $\epsilon_{\text {coupled }}=50 \hbar$. A coupled energy resolution as low as $27 \hbar$ has been recently obtained with a two-stage SQUID amplifier coupled with an electrical resonator and cooled down to $T=50 \mathrm{mK}$ [20]. The spherical GW antenna Minigrail and the AURIGA detector are currently operating at $5 \mathrm{~K}$ with SQUID amplifier energy resolution of the order of $600 \hbar$ [1, 21]. More than a factor of 10 improvement is expected when this detector will operate at $T<100 \mathrm{mK}$.

From the plots in figure 4 we see that, in terms of noise temperature and bandwidth, a large mass spherical detector developed using the available technology will not perform better than an ultra-cryogenic bar detector operating at the same frequency. As a matter of fact, the noise temperature $T_{N}$ and the bandwidths depend only on the frequency, the electro-mechanical impedance matching and the SQUID amplifier noise. However, due to the larger cross section of a spherical detector, with respect to a bar detector at the same frequency, the spherical detector improves the SNR by a factor of about 40 [12]. Moreover, 

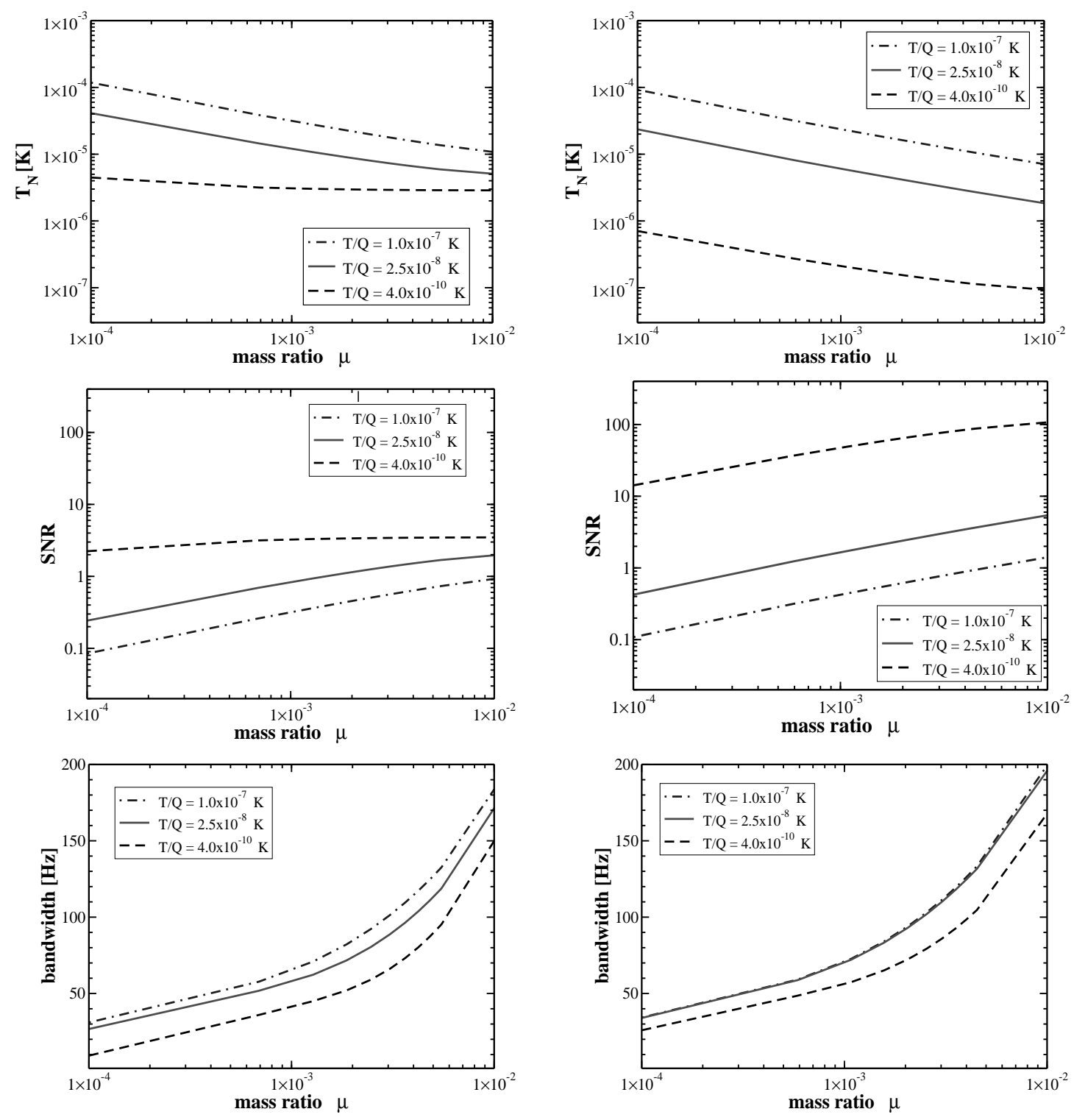

a)

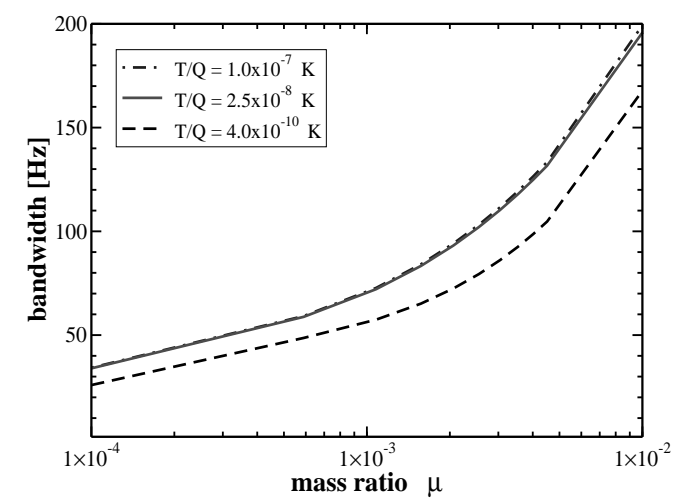

b)

FIG. 4: Noise temperature, SNR and bandwidth of the spherical detector as a function of the resonator mass ratio $\mu$. The detector is read-out by a $50 \hbar$ energy resolution $(a)$ and quantum limited (b) SQUID amplifier. Three different configurations with $T / Q=1 \cdot 10^{-7} K, 2.5 \cdot 10^{-8} K$ and $4 \cdot 10^{-10} \mathrm{~K}$ are studied. The SNR is calculated for a $T_{G B}=10 \mu \mathrm{K}$ gravitational wave burst.

omnidirectionality will of course still be a unique feature of a spherical detector when at least six resonators are used. With $T / Q=2.5 \cdot 10^{-8} K$ and mass ratio $\mu=0.001$, corresponding to a transducer resonating mass of about $9 \mathrm{Kg}$, a capacitance of $10 \mathrm{nF}$ and an optimal electrical field $E=5 \cdot 10^{6} \mathrm{~V} / \mathrm{m}$, the detector has a noise temperature $T_{N}$ of about $10 \mu \mathrm{K}$, about a factor 
of 30 better than the present most sensitive resonant bar detector [1].

The SQUID amplifier is considered strongly coupled to the electrical matching circuit assuming $k=0.8, L_{p} \sim 1 H$ and $L_{s} \sim L_{i}=1.7 \mu H$. As in bar detectors, the sensitivity will be mainly limited by thermal noise of the electrical resonator, and, outside the resonances, by the SQUID amplifier additive noise. The curves in figure 4, a clearly show that large mass ratio brings benefits only to the bandwidth and not to the detector noise temperature. This is due to the fact that a large mass resonator will not help to decrease the thermal noise contribution. As a matter of fact, the contribution of the mass at the denominator of the Langevian forces derived in Eqs. (34) is almost cancelled by the transfer function when transforming the forces into displacement. On the other end, the bandwidth will increase for large mass ratio due to a better impedance matching. The latter will unavoidable enlarge the contribution of the back action noise coming from the SQUID amplifier producing the observed saturation in the detector sensitivity at large mass ratio.

To fully exploit the potentiality of a massive spherical detector one needs to develop capacitive transducers with high sensitive area, massive mechanical resonators with Q-factors larger than $10^{7}$, high Q electrical transformers and large bias electric fields.

In figure 4.b the detector sensitivity is given for the antenna and transducer chain parameters obtainable by pushing the current technology to its limits. We consider a quantum limited SQUID amplifier operating at temperature $T<100 m K$. Again we estimate the noise temperature $T_{N}$, the bandwidth and the SNR for a $10 \mu K$ burst for $T / Q=1 \cdot 10^{-7} K, 2.5 \cdot 10^{-8} K, 4 \cdot 10^{-10} K$. By comparing those plots with the ones previously discussed, it becomes clear that without an improvement of the mechanical and electrical resonators with respect to available technology, the use of a quantum limited SQUID amplifier will not benefit the detector sensitivity. By looking at the dot-dashed curves of figure 4 , for examples, corresponding to a $T / Q=1 \cdot 10^{-7} \mathrm{~K}$, one shall expect no improvement in the strain sensitivity, but only a small increase of the bandwidth due to the lower additive noise of the SQUID. A spherical detector operating at $T=20 \mathrm{mK}$ with mechanical and electrical quality factor $Q \sim 5 \cdot 10^{7}$, a quantum limited SQUID amplifier and large mass mechanical transducers, $\mu=0.01$ and $m_{R}=90 \mathrm{Kg}$, can have a noise temperature of $1.3 \cdot 10^{-7} \mathrm{~K}$, corresponding to a peak strain sensitivity of $10^{-23} H z^{-1 / 2}$ at $1 k H z$, and a bandwidth of about $200 \mathrm{~Hz}$. This would improve of a factor 50 the sensitivity of an existing bar or small sphere antenna working at the quantum limit. The bandwidth will have only 
a moderate increase with respect to present resonant bar antennae [1], merely due to the spreading of the spheroidal modes. The minimum achievable antenna noise temperature is given by the quantum-mechanical limit of a linear motion detection derived by Giffard [43] and equal to $T_{N, \min }=2 \hbar \omega / k_{B}\left[\exp \left(\hbar \omega / k_{B} T_{N, a}\right)-1\right]$, where $T_{N, a}$ is the noise temperature of the linear amplifier. When a quantum limited amplifier is used, $T_{N, \min }=9.5 \cdot 10^{-8} \mathrm{~K}$ for a $\mathrm{kHz}$ resonant sphere considered here.

\section{B. Strain sensitivity}

We show here the strain sensitivity calculated using Eqs. (26). The strain curves are derived for each noise contribution described in section III. The read-out circuit was optimized as discussed above.

In figure 5 the strain sensitivity is calculated for a spherical detector equipped with a single transducer placed in the position 1. It was calculated for an optimally oriented source, as will be described in detail below. The detector parameters used for this simulation have already been achieved in separate experiments [1, 2, 3]. The sensitivity curves are obtained considering the detector operating at $T=50 \mathrm{mK}$ with a $50 \hbar$ SQUID amplifier and with the electrical mode coupled to the mechanical ones. The quality factors are chosen to be $Q=3 \times 10^{6}$ and $Q=2 \times 10^{6}$ for the mechanical and electrical modes respectively. The mass ratio is $\mu=0.001$, corresponding to a mechanical resonator mass $m_{R} \simeq 9 \mathrm{Kg}$. The optimal electrical bias field is about $E=5 \cdot 10^{6} \mathrm{~V} / \mathrm{m}$ and the transducer capacitance is $10 \mathrm{nF}$.

The strain sensitivity shown in figure 6 was calculated for a spherical detector with six transducers in the TI arrangement. The detector and transducers parameter are the same used in the single transducer configuration described above. Differently from the single transducer configuration, the sensitivity is independent of the wave direction and polarization. In such a configuration the detector sensitivity will be limited by the thermal noise contribution from the mechanical and electrical resonators and by the back action noise of the SQUID amplifier due to the the optimal matching coming from the tuning of the electrical resonances with the mechanical ones. Outside the resonances, the sensitivity is limited by the additive current noise of the SQUID amplifier.

In figure 7 the strain sensitivity is shown for an optimized detector equipped with SQUID amplifiers operating at the quantum limit. The simulation considers a detector operating 


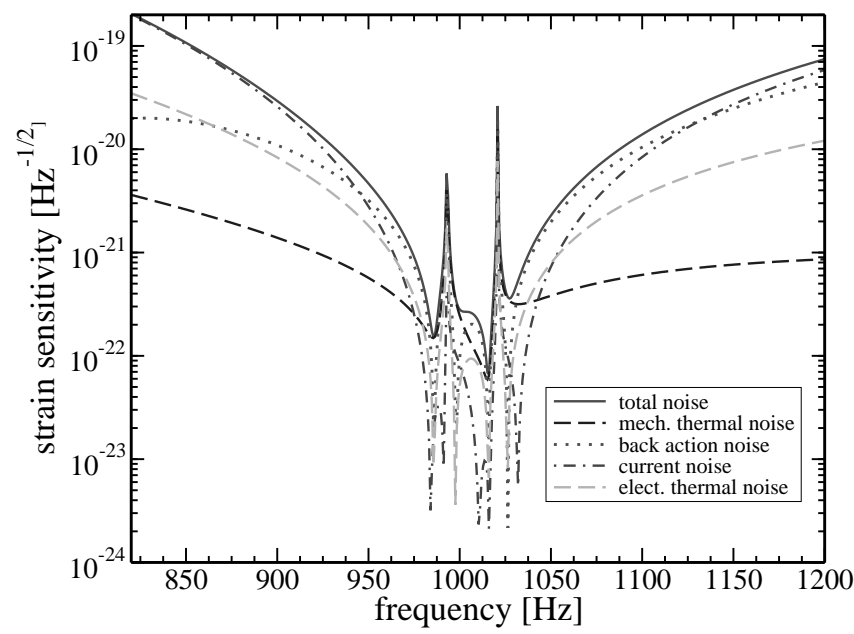

FIG. 5: Strain sensitivity for a spherical detector with one single transducer, $T / Q \sim 2.5 \times 10^{-8}$, resonators mass ratio $\mu=0.001$ and 50 energy resolution SQUID amplifiers. The transducer electrical mode is tuned to the mechanical ones. The parameters used for this simulation have already been achieved in separate experiments with bar detectors or a lower mass spherical antenna.

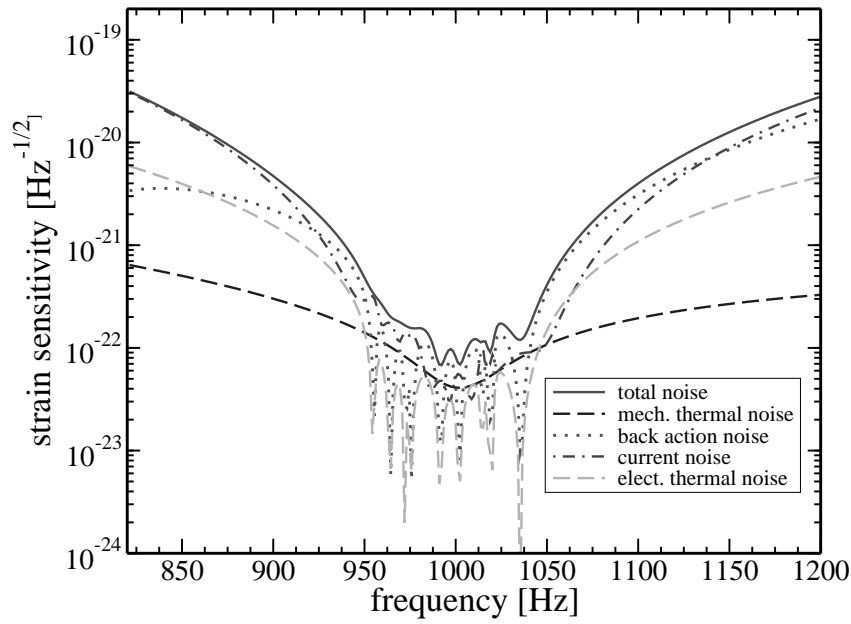

FIG. 6: Strain sensitivity for a spherical detector with six transducers, $T / Q \sim 2.5 \times 10^{-8}$, resonators mass ratio $\mu=0.001$ and $50 \hbar$ energy resolution SQUID amplifiers. The electrical modes are tuned to the mechanical ones. The parameters used for this simulation have already been achieved in separate experiments with bar detectors or lower mass spherical antenna.

at $T=20 \mathrm{mK}$ with all resonators having quality factors $Q=5 \times 10^{7}$. We consider six transducers in the TI arrangement. The mass ratio is $\mu=0.01$ and the optimal electrical bias field is $E \sim 3 \cdot 10^{7} \mathrm{~V} / \mathrm{m}$. We consider a transducer with a large capacitance of about 


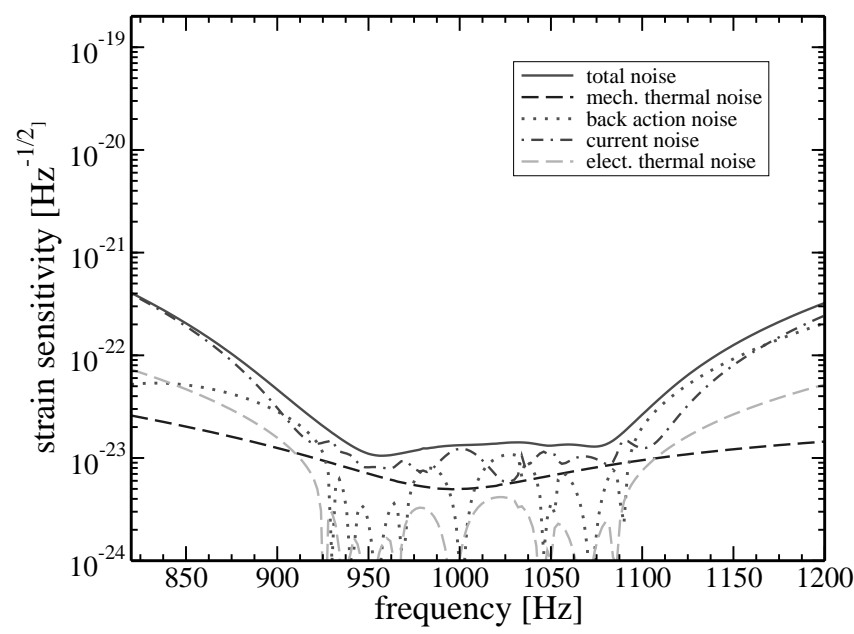

FIG. 7: Strain sensitivity for a spherical detector with $T / Q=4 \times 10^{-10}$, resonators mass ratio $\mu=0.01$ and $1 \hbar$ energy resolution SQUID amplifiers. The electrical modes are tuned to the mechanical ones.

$C=30 n F$. The sensitivity is mainly limited by additive and back action noise of the SQUID linear amplifiers, whose minimum energy resolution is imposed by quantum mechanics. Despite the low working temperature and the high Q considered, a small contribution from the thermal noise is still present.

\section{Antenna pattern}

We calculate here the SNR of a spherical detector equipped with one, three and six resonators as a function of the direction and polarization of the incident wave. We consider a detector operating at nearly the quantum limit. We numerically calculate the SNR as a function of the three Euler angles $(\psi, \theta, \phi)$ of Eqs. (7). All the three angles are necessary to completely define the GW source. The first Euler angle $\psi$ carries information about the wave polarization [44]. The other two, $(\theta, \phi)$, give the source direction. In the sky maps presented in this section, we call those angles respectively declination $\delta=\pi / 2-\theta$ and right ascension $\alpha=\phi$ to match the astronomical notation. The antenna patterns depend on the longitude and the latitude of the detector location as well as on the Universal Time (UT), $\tau$, due to the earth proper rotational motion. To simplify the discussion we consider here detectors at $\tau=0$. We calculate the antenna patterns for two spherical detectors, $2 \mathrm{~m}$ in diameter, located at Leiden (The Netherlands), lon $=4^{\circ} 30^{\prime \prime}$, lat $=52^{\circ} 7^{\prime \prime}$, and at Saõ Paulo 

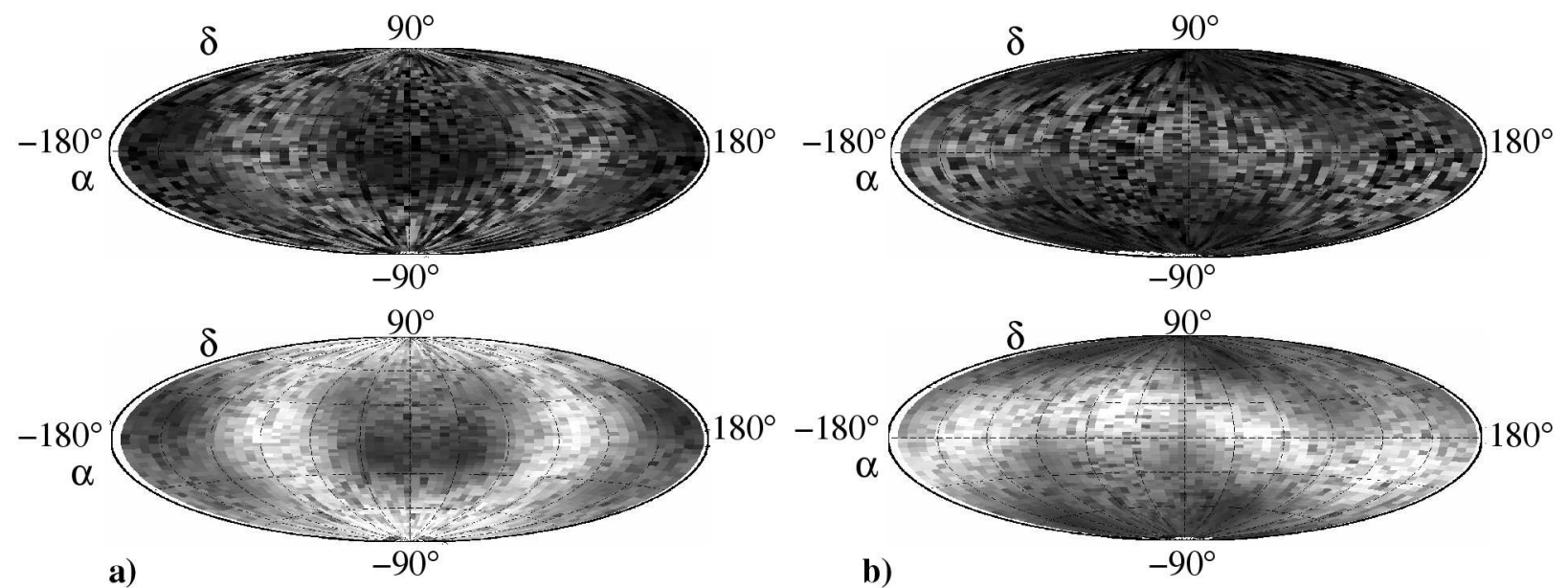

FIG. 8: Antenna pattern for spherical detectors respectively located in Leiden a) and Saõ Paulo b) with a single transducer on position 1 of the six positions of the TI arrangement. We consider a linearly polarized (top) and a one-cycle, circularly polarized (bottom) signal as explained in the text, depositing an energy $T_{G B}=3 \mu K$ in the detector.
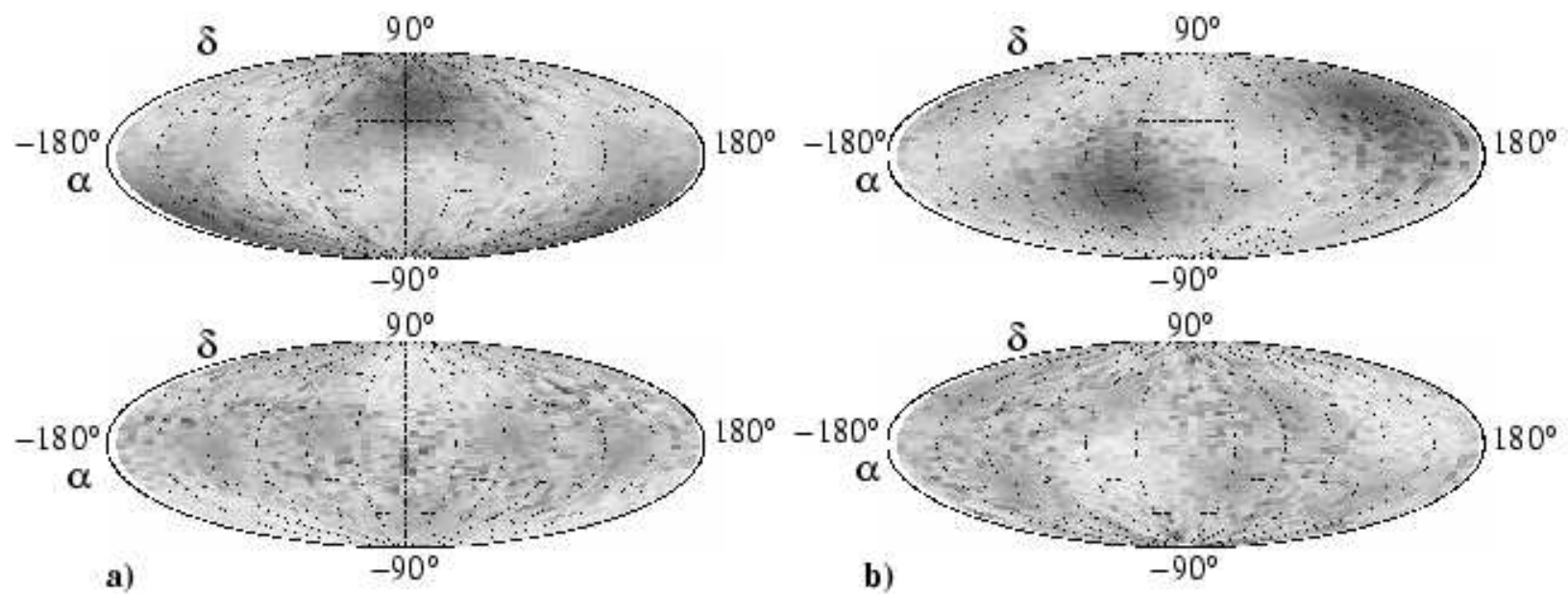

FIG. 9: Antenna pattern of spherical detectors respectively located in Leiden a) and Saõ Paulo b) with a three transducers set in the TI configuration at $\theta_{T I}=37.3773^{\circ}$ (sky maps above) and $\theta_{T I}=78.1876^{\circ}$ (sky maps below). We consider a one-cycle circularly polarized sinusoidal signal depositing an energy $T_{G B}=3 \mu K$ in the detector

(Brasil), lon $=-46^{\circ} 38^{\prime \prime}$, lat $=23^{\circ} 34^{\prime \prime}$, where respectively the MiniGRAIL and the Mario Schemberg, $65 \mathrm{~cm}$ large in diameter, spherical detectors are being developed. We chose those locations because the general discussion about direction sensitivity is independent on the detector size and mass a part from the absolute value of SNR and bandwidth. The 
analysis presented here is then useful for the existing small spherical detectors as well.

In figure 8 the sky maps are shown for a spherical detector with a single transducer on position 1 of the TI arrangement. We consider a linearly polarized wave with $h_{+}=h_{0}$ and $h_{\times}=0$ and a random polarization angle $\psi$ (skymaps at top). The amplitude $h_{0}$ is related to the deposited energy $E=k_{B} T_{G B}$ according to Eqs. (311). For each simulation we indicate the GW energy in the figure captions. The SNR for each wave direction depends on the polarization angle. The choice of a random polarization for each direction trial produces the scattered pattern. When a circularly polarized wave is chosen (skymaps at bottom) the maps become smoother and the SNR is only dependent on the wave direction. Here and in the following we consider a one-cycle, circularly polarized sinusoid: $h_{+}=h_{0} \sqrt{2} \cos (\omega t)$ and $h_{\times}=h_{0} \sqrt{2} \sin (\omega t), 0 \leq \omega t \leq 2 \pi$, with the frequency $\omega$ laying within the detector bandwidth. Transducers on other positions will show a similar pattern rotated of proper angles accordingly to the positions. The sky maps on the left (figure 8, a) refers to a detector in Leiden with the lab-frame oriented so that the z-axis is pointing to the local vertical and the $\mathrm{x}$ axis to the local south. The sky maps on the right (figure 8, b) refers to the a detector in Saõ Paulo. As expected, in a spherical detector operating with only one transducer the sensitivity is direction and polarization dependent and changes according to the transducer location. A source emitting a linearly or circularly polarized wave is more likely to be detected when laying on the plane perpendicular to the transducer axis and passing through the sphere center.

In figure 9, the sky maps are shown for a spherical detector with three transducers placed at the three positions of the TI arrangement, respectively at $\theta_{T I}=37.3773^{\circ}$ and $\theta_{T I}=78.1876^{\circ}$. The signal used for the simulation is a one-cycle circularly polarized sinusoid with energy $T_{G B}=3 \mu K$. The higher maximum SNR with respect to the single transducer configuration is mainly due to the large detector bandwidth. When six identical resonators are used, the detector becomes independent form the wave incoming direction and polarization [44]. This result is also shown in figure 10. For a real detector with identical transducers, not optimally tuned to the 5 spheroidal modes, a small residual anisotropy in the SNR and bandwidth is still present. This is however less than $10 \%$ over the whole sky. The signal bandwidth anisotropy is shown in figure 11, Both the figures will be further discussed in the following section.

Figure 12 presents the detection efficiency of a spherical detector equipped with only one 


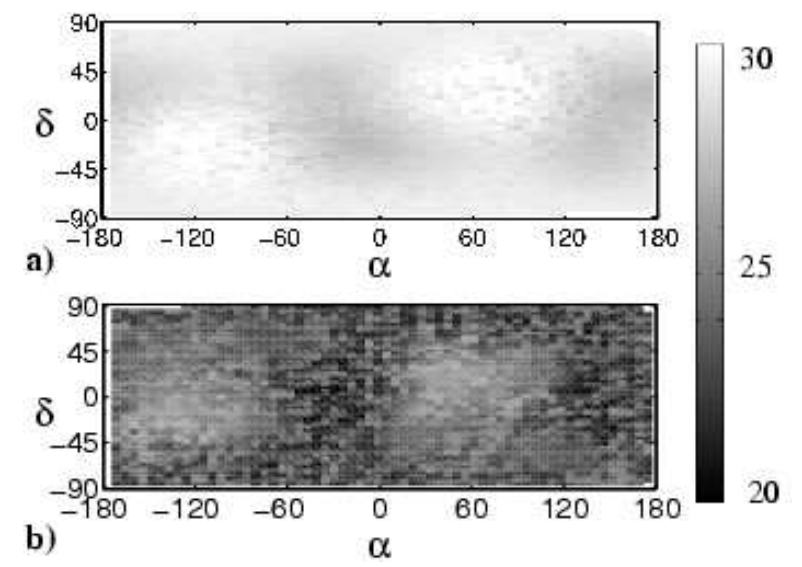

FIG. 10: SNR of a quantum limited spherical detector with six transducers in the TI configuration. In a), identical transducers are considered. In b), we arbitrary modified the parameters of each transducer of a maximum of $15 \%$ from their optimal value. We consider a one-cycle circularly polarized wave with energy $T_{G B}=3 \mu \mathrm{K}$.

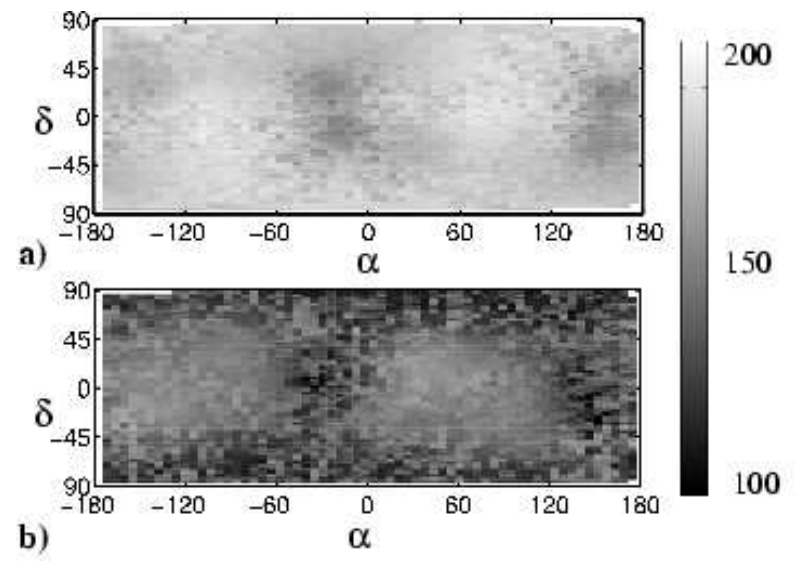

FIG. 11: Signal bandwidth of a quantum limited spherical detector with six transducers in the TI configuration. In a), identical transducers are considered. In $\mathbf{b}$ ), we arbitrary modified the parameters of each transducer of a maximum of $15 \%$ from their optimal value. We consider a one-cycle circularly polarized sinusoidal signal with energy $T_{G B}=3 \mu \mathrm{K}$.

transducer as a function of the GW incoming direction for different GW energies, $T_{G B}=$ $1,1.5,3 \mu K$ equal to a maximum SNR respectively of $\rho_{\max } \sim 10,15,30$. In analogy with [45], the detection efficiency is defined as $\frac{1}{2} \operatorname{erfc}\left[\left(\eta-\rho_{0}\right) / \sqrt{2}\right]$, where $\eta$ is a threshold chosen equal to 5 and $\operatorname{erfc}$ is the complementary error function. The detection of a one-cycle circularly polarized wave of energy $T_{G B}=3 \mu \mathrm{K}$ is almost always succesfull independently 


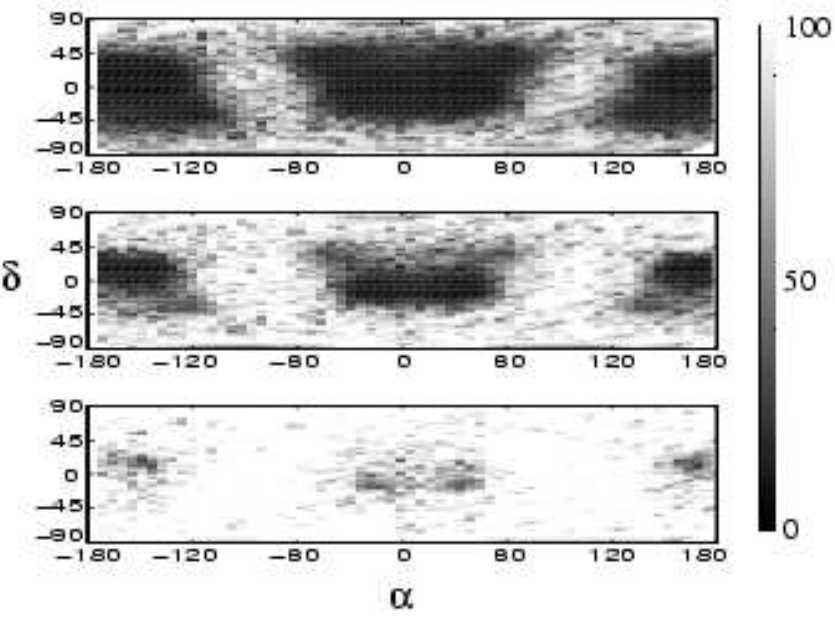

FIG. 12: Comparison of detection efficiency sky maps for a spherical detector with one transducer and for different deposited energy $T_{G B}=1,1.5,3 \mu K$ (from top to bottom). We consider a circularly polarized wave as described in the text.

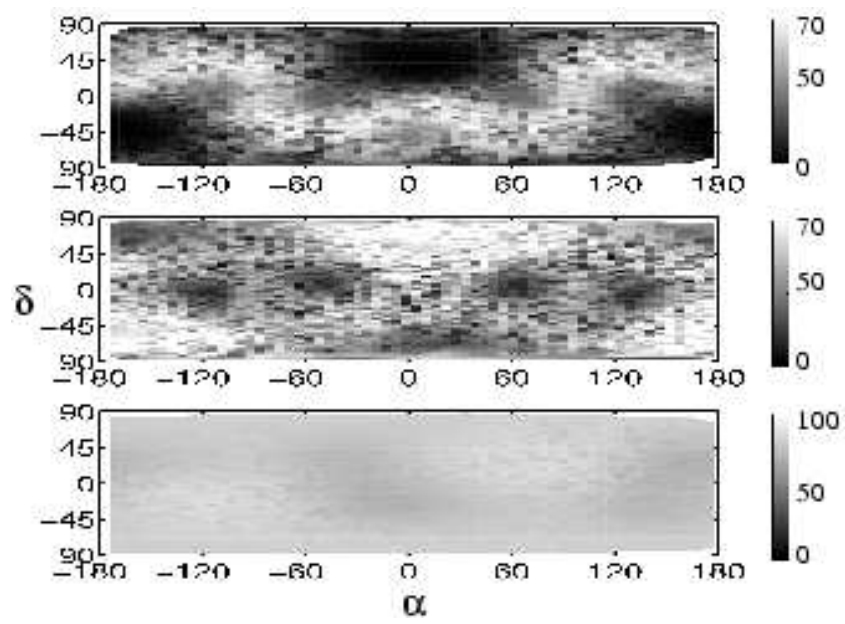

FIG. 13: Comparison of detection efficiency sky maps for a spherical detector with three transducers in the TI positions respectively at $\theta_{T I}=37.3773^{\circ}$ and $\theta_{T I}=73.1876^{\circ}$, and for a complete detector with 6 transducers (from top to bottom). We consider a GW burst deposited energy of $0.6 \mu \mathrm{K}$ and a circularly polarized wave. Note the difference in the color code on the graphs and that the signal amplitude is smaller than the ones in figure 12 .

of the incoming direction. In figure 13 similar skymaps are shown for a $T_{G B}=0.6 \mu \mathrm{K}$ when a spherical detector with 3 and 6 transducers is considered. The results of the skymaps in figure 12 are summarized in figure 14 where the fraction of sky is plotted as a function of the detection efficiency for a single transducer configuration and different signal amplitudes. 


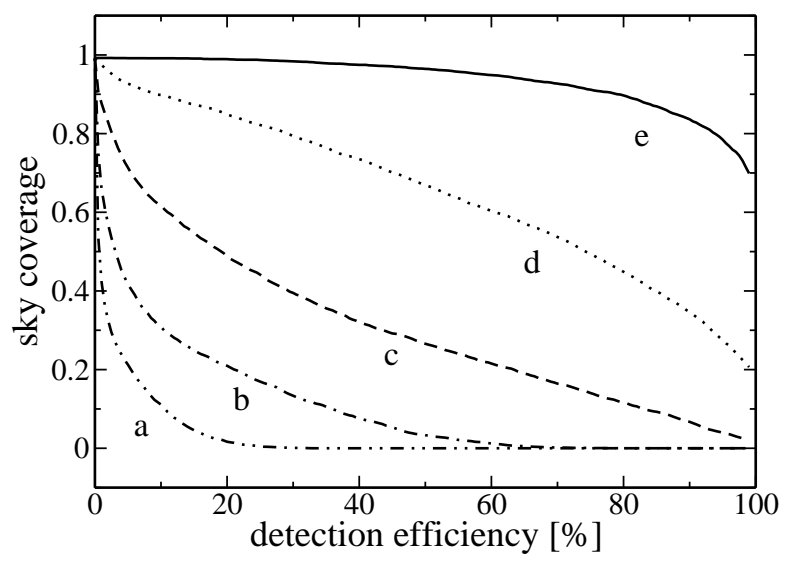

FIG. 14: Fraction of sky as a function of detection efficiency for a detector with a single transducer. We consider a circularly polarized wave with $T_{G B}=0.6,0.75,1,1.5,3 \mu K$, and $\rho_{\max } \sim 6,7.5,10,15,30$, respectively denoted by $\mathbf{a}, \mathbf{b}, \mathbf{c}, \mathbf{d}$ and $\mathbf{e}$.

One notes that the fraction of sky covered decreases when the detection probability level increases: the curves evolution for each SNR can be understood from the patterns of the skymaps in figure 12. The detection probability is higher than $30 \%$ in $40 \%$ of the sky for a $\rho_{\max }=10$, corresponding to a $1 \mathrm{~ms}$ GW burst of amplitude $h_{0}=2 \cdot 10^{-21}$; it is $50 \%$ for almost a $70 \%$ fraction of the sky when $\rho_{\max }=15$. For $\rho_{\max } \simeq 30$ the detection is likely almost in any direction.

Figure 15 presents the fraction of sky as a function of the detection efficiency of a GW burst of deposited energy $T_{G B}=0.6 \mu \mathrm{K}$ for different transducer configurations. Plot i) refeers to a single spherical antenna, while plot ii) shows the detection efficiency of two optimally oriented spherical detectors located at Leiden and Saõ Paulo. This was obtained by maximizing the portion of sky simultaneously seen by both detectors as a function of the orientation of the single detector reference system with respect to the local south. This corresponds to a rotation of the brasilian detector lab frame $(x, y, z)$ (see figure 11) around the local z-axis of about $\phi_{0}=-135^{\circ}$.

A detector with a single trasducer is unable to detect any signal from the sky when the SNR is as low as $\rho_{\max }=6$. For the same SNR, the sky coverage of a single detector with three and six transducer is respectively more than $40 \%$ and $100 \%$ with $50 \%$ detection efficiency. This is mainly due to the increase of detector bandwidth when more than one transducers are used. Figure 15,ii shows that for detector with one or three transducers, 
a) single detector

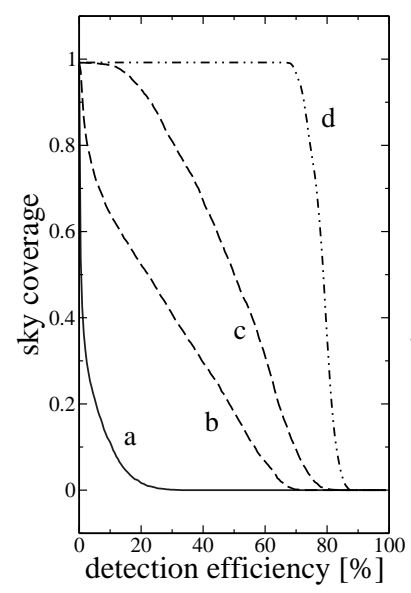

b) two detectors

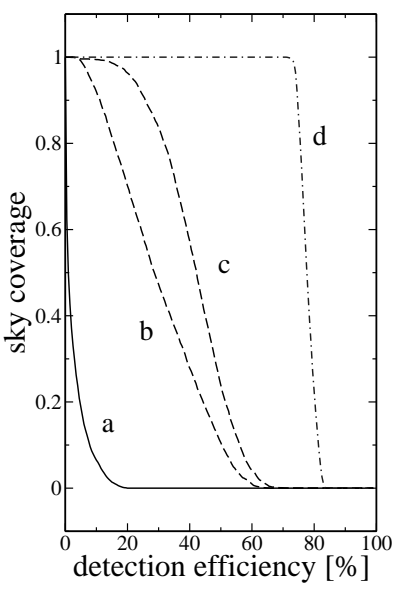

FIG. 15: Fraction of the sky as a function of the detection efficiency for a GW burst of deposited energy $T_{G B}=0.6 \mu \mathrm{K}$ and $\rho_{\max }=6$ for a single spherical antenna i) and two optimally oriented spherical detectors ii) located at Leiden and Saõ Paulo. Curve a corresponds to a read-out configuration with a single transducer located in position 1 of each detector, curves $\mathbf{b}$ and $\mathbf{c}$ to a three transducers configuration with $\theta_{T I}=37.3773^{\circ}$ and $\theta_{T I}=73.1876^{\circ}$ and curve $\mathbf{d}$ to a complete spherical detector with 6 transducers.

the twofold coincidence probability remains lower than the detection efficiency of a single sphere. Two spherical antenna with six transducers can equally detect any sources in the sky even when the SNR is as low as $\rho_{\max }=6$.

\section{Sensitivity of a sphere with not ideal transducers}

It has been shown that for a perfect sphere the resonators mistuning and misplacing has little effect on the isotropy when the deviation from the ideal TI configuration is less than $1 \%$ [13, 44]. We study here two other possible degradation effects which may arise in a real spherical detector. The first is related to the broken spheroidal mode degeneracy and the second to the the fact that a real transducer is not a pointlike mass as generally considered. In a real detector the spheroidal mode degeneracy is broken due to the suspension and the holes made on its surface to house the transducers. The modes spreading can be as large as $5 \%$ of the main resonance. It becomes natural to ask to which modes each transducer should be tuned and how good the tuning should be to avoid a sensitivity degradation. We 
consider here a numerical analysis of the transducer mechanical and electrical mistuning for a sphere with six resonators with a mass ratio $\mu=0.01$ and operating at nearly the quantum limit.

In figure [16 $\mathrm{a}$ ), we see the effect on the SNR for a $T_{G B}=3 \mu \mathrm{K}$ circularly polarized GW burst when the natural resonances of each resonator are modified from the initial values $\omega_{0}=2 \pi[987,1001,1001,1008,1012,1017]$, arbitrarily chosen equal to each of the spheroidal mode resonances. One finds a maximum change of $10 \%$ in the SNR for a resonator mistuning of about $10 \%$. It is possible to optimize the tuning by shifting the resonator resonance frequency as much as indicated by the maximum of the $S N R$ in figure 16. a. This procedure can be repeated several times. The result is shown in figure 16, b, where the SNR is maximum for each resonator around the new set of the resonators natural frequencies $\omega_{1}=2 \pi[1047,971,980,970,960,1027]$. Such an optimal frequency set derives from a combination of multiple coupling, modes splitting and transducers position. Once the bare sphere spheroidal modes and the resonator masses and positions are known, one can always find an optimal natural resonance for each resonator.

We remark here that, due to the presence of so many modes and the related multiple splitting, it could be difficult in practice to determine the resonators frequency with an accuracy better than $10 \%$ and one has probably to accept a not optimized detector. The loss in sensitivity is in any case less than $10 \%$ for a $100 \mathrm{~Hz}$ mistuning of the mechanical modes of a detector resonating at $1 \mathrm{kHz}$ and with a tuned matching network.

So far we consider the transducers operating on the sphere all identical except for their main resonance frequency. Here we evaluate the effect on the detector anisotropy in the sensitivity and bandwidth, which derives from using six not identical transducers to readout the five quadrupolar mode of the sphere. We arbitrary modified the parameters of each transducer such as mass, mechanical and electrical quality factor, transducer capacitance, electrical coupling factor and SQUID noise of a maximum of $15 \%$ from their optimal value. As shown in figure 10, b, the detector SNR, for a circularly polarized GW burst with $T_{G B}=$ $3 \mu K$, is reduced about the $30 \%$. This is mainly due to a decrease in signal bandwidth as one can see from figure 11,b.

In the following we study how the detector sensitivity decreases when the transducer electrical resonator is not perfectly matched to the mechanical resonator. Such a situation could arise in practice when the electrical mode cannot be arbitrarily adjusted to the opti- 


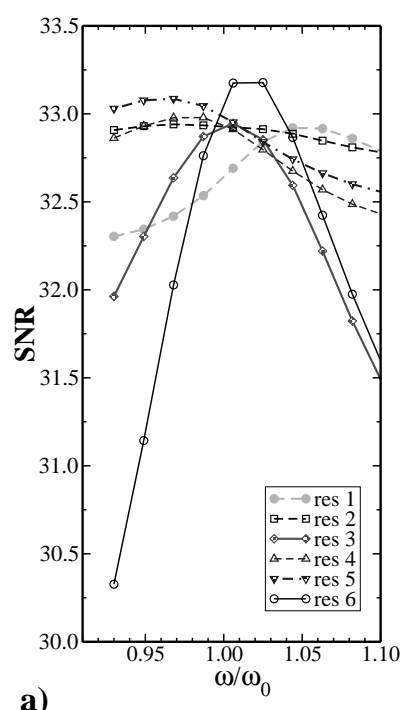

a)

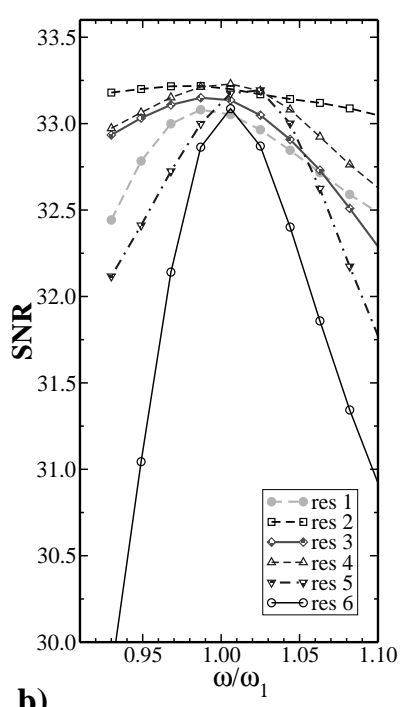

b)

FIG. 16: Resonators mistuning. The graph shows the detector SNR for a $T_{G B}=3 \mu K$ one-cycle circularly polarized GW burst as a function of the mistuning parameter $\omega / \omega_{0}$ for each resonator in the case of a) a starting arbitrary set of natural resonances $\omega_{0}=2 \pi[987,1001,1001,1008,1012,1017]$ and b) an optimized set $\omega_{1}=2 \pi[1047,971,980,970,960,1027]$ obtained after few tuning iterations.

mum value define by Eqs. (38) as a consequence, for example, of a voltage leakage in the bias lines. One finds that, when the electrical mode of only one transducer is mistuned, even up to about $30 \%$ of the optimal frequency, very little effect is observed in the SNR and bandwidth. This is evident from the full circle data of figure 17, where the detector SNR and bandwidth for a $T_{G B}=3 \mu \mathrm{K}$ circularly polarized $\mathrm{GW}$ burst is given as a function of the mistuning parameter $\omega / \omega_{e l}$, being $\omega_{e l}$ equal to the optimized mechanical resonator frequency set derived above. When all the electrical modes are decoupled from the mechanical modes, the SNR and bandwidth decrease of a factor proportional to the mistuning factor as shown by the open squared data of figure 17.

In the numerical analysis of resonant detectors one always considers the mechanical resonators as point-like masses. A real transducer is sampling the sphere surface radial displacement in many points belonging to the contact surface between the resonator spring and the sphere. The actual displacement can be seen as the results of an average of such a sampling. We calculated here the detector sensitivity when a real resonator is considered. Three kinds of resonators are generally used in resonant detectors: mushroom, membrane and "rosette" resonators. All of them have in common the fact that the spring is attached 

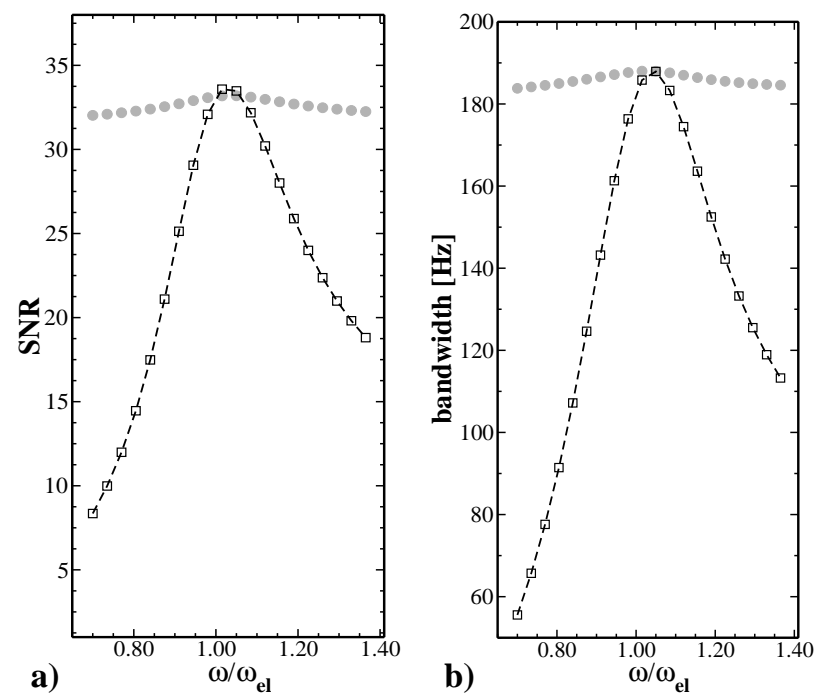

FIG. 17: Mistuning of the electrical mode. The graph shows the detector SNR (a) and bandwidth (b) for a $T_{G B}=3 \mu K$ one-cycle circularly polarized GW burst as a function of the mistuning parameter $\omega / \omega_{e l}$ where $\omega_{e l}$ is equal to the optimized mechanical resonator frequency set. The data show the effect when the electrical mode of only one transducer is detuned (full circles) and when the electrical modes of all the six transducers are equally mistuned (open squared).

to a support, which is rigidly connected to the antenna's surface. The variable read-out considered in our calculation, in analogy with [34], can be written as

$$
x(t)=\int f\left(\mathbf{r}_{\mathbf{t}}, \mathbf{r}_{\mathbf{r}}\right) y\left(\mathbf{r}_{\mathbf{t}}, \mathbf{r}_{\mathbf{r}}, t\right) d r_{t} d r_{r}
$$

where $y(\mathbf{r}, t)$ is the radial displacement of the intersection points between the support and the springs of the mechanical resonator used to amplify the sphere displacement. $f(\mathbf{r})$ is a form factor and the integral is calculated along the resonator support-spring intersection. We consider transducers with cylindrical symmetry where $r_{t}$ is the radius of the supportspring intersection and $r_{r}$ gives the radial position of the intersection points referred to the sphere centre. For "mushroom", "rosette" and "membrane" resonators those intersection are, respectively, the "mushroom" leg section, the cylindrical section where the membrane is attached to the resonator ring support, and the "rosette" spring sections at the attachment point with the ring support.

The form of the radial displacement depends strongly on the resonator geometry and springs topology, making it difficult to find an analytical expression like the one for the gaussian laser beam considered in [34], and [35]. In the case when only the first spheroidal 
modes are considered, the form factor for a "membrane" and "rosette" transducer becomes

$$
f_{m e m b}\left(\mathbf{r}_{\mathbf{t}}, \mathbf{r}_{\mathbf{r}}\right)=\frac{s}{2 \pi r_{t} \delta r_{r}} \frac{A_{12}\left(r_{r}\right)}{A_{12}\left(r_{s}\right)}
$$

where $\delta r_{r}$ is the spring thickness and $A_{12}(r)$ is the spheroidal quadrupole radial amplitude function described in [11]. For a "membrane" transducer $s=1$, while for a "rosette"

transducer $s=S_{\text {ros }} / S_{\text {memb }}<1$ is the ratio between the intersection surfaces defined by the "rosette" springs and a membrane of the same thickness.

In the case of a "mushroom" transducer, we have

$$
f_{\text {mush }}=\frac{1}{\pi r_{t}^{2}} \frac{A_{12}\left(r_{r}\right)}{A_{12}\left(r_{s}\right)} .
$$

In proximity of the sphere surface the radial amplitude is slowly changing and the ratio $A_{12}\left(r_{r}\right) / A_{12}\left(r_{s}\right)$ can generally be approximated to 1 .

In figure 18 the SNR and bandwidth for a circular polarized $T_{G B}=3 \mu \mathrm{K} \mathrm{GW}$ burst of a nearly quantum limited sphere with six resonators is shown as a function of the resonator radius $r_{t}$. The improvement in sensitivity, obtainable as described above by using massive resonator, with $\mu=0.01$, is slightly reduced by the resonators large radius $r_{t} \sim 0.2 m$. The SNR decreases of about $10 \%$ in this case. A "mushroom" resonator is preferable, as shown in figure 18, but high $\mathrm{Q}$ massive resonators are difficult to achieve for such a geometry. In the sky maps shown in figure 19, obtained from 1000 randomly distributed events of circularly polarized GW bursts, the SNR and bandwidth anisotropy for a transducer with $r_{t}=0.2 \mathrm{~m}$ is shown. One finds up to $15 \%$ of asymmetry in the SNR and up to $40 \%$ in the detector bandwidth.

\section{CALIBRATION OF A SPHERICAL GRAVITATIONAL WAVE DETECTOR}

To calibrate a gravitational wave detector one needs to postulate a model of the complete detector whose parameters are experimentally determined. The calibration is performed in two steps. First the effective temperature of the modes is estimated. This is important in order to understand the detector dynamics and to estimate the noise contributions. Secondly, one has to measure the detector response to an applied force, which is equivalent to evaluating the transfer function $\mathbf{G}_{\mathbf{s i g}, \mathbf{I}}$ in Eqs. (26) . We stress here that a full antenna characterization implies the estimation of the terms of the matrix $\mathbf{G}_{\mathbf{s i g}, \mathbf{I}}$, which relates the input 

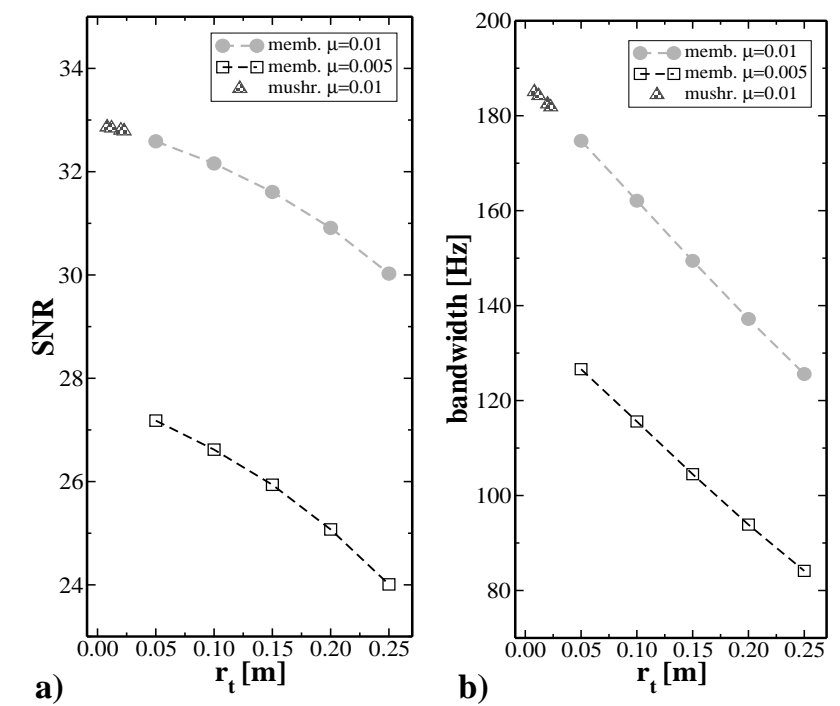

FIG. 18: Detector $S N R$ and signal bandwidth as a function of the resonator radius $r_{t}$ for a "membrane" resonator with mass ratio $\mu=0.005$, open squares, and $\mu=0.01$, full circles, and a "mushroom" resonator with $\mu=0.01$, open triangles.

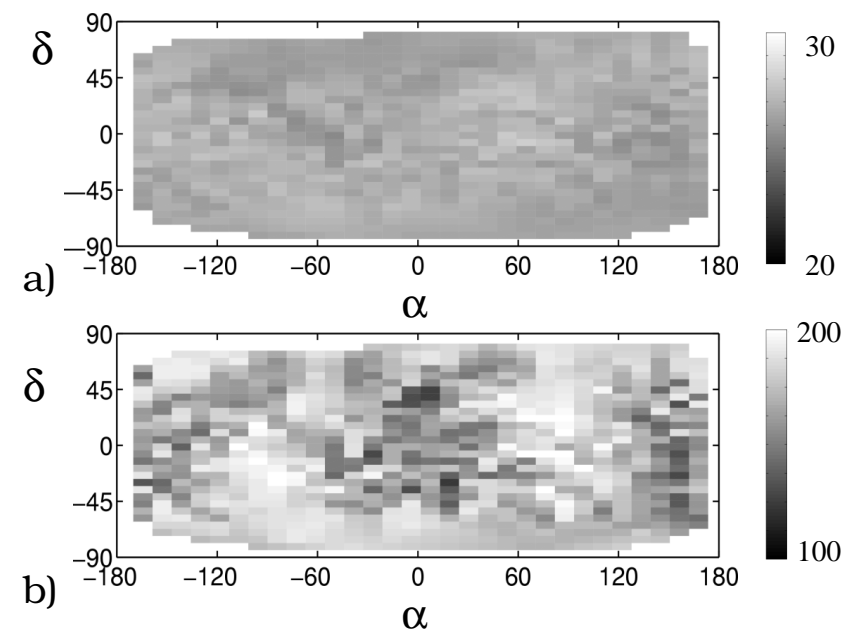

FIG. 19: Detector $S N R$ and signal bandwidth anisotropy for a circular polarized GW burst with $T_{G B}=3 \mu K$ when a "rosette" resonator with $r_{t}=0.2 \mathrm{~m}$ is used to read out the sphere quadrupolar modes.

signal and noise to each transducer SQUID input current. It can be achieved by injecting a known signal at each step of the transducer chain, i.e: radial forces to the five spheroidal modes of the sphere, radial forces to each resonator, voltage signal in the superconducting transformer and voltage signal at the SQUID input. In order to evaluate the sensitivity of 
the antenna to GW signal, however, one needs especially to measure the transfer function which converts the forces applied to the spheroidal modes to the SQUID input current of each transducer. By measuring the noise of each transducer during normal operation, one can finally estimate the strain sensitivity as in Eqs. (26) .

We describe below two methods that can be used to perform a complete calibration of a spherical detector in analogy with the method used for the bar detector AURIGA [1, 23]

\section{A. Modes equivalent temperature}

According to the fluctuation dissipation theorem, the voltage and current power spectra observed in an electrical circuit, when only thermal noise sources are presents, are given by

$$
S_{V}=4 k T R e(Z(\omega)), \quad S_{I}=4 k T R e\left(\frac{1}{Z(\omega)}\right),
$$

where $Z(\omega)$ is the impedance seen at the input of the amplifier used for the read-out and $\mathrm{T}$ is the thermodynamic temperature. By measuring the transducer output spectrum in normal operation and the input impedance of the circuit one can evaluate the equivalent temperature of the transducer chain. If only thermal noise is present, the equivalent temperature of the chain should be equal to the thermodynamic temperature of the experiment. Because the SQUID is not an ideal amplifier, its current and voltage noise give a contribution to the total transducer output noise. This contribution is in general not negligible. The total current noise measured at the input of each transducer line SQUID can be written as [1],

$$
S_{I}=4 k T \frac{Q_{a}}{Q_{e l}} R e\left(\frac{1}{Z(\omega)}\right)+\frac{S_{v v}(T)}{|Z(\omega)|^{2}}+S_{I i i}(T),
$$

where the second term on the right hand side of the equation is the back action noise contribution from the SQUID amplifier with $S_{v v}$ given by Eqs. (36). $Q_{a}$ is the apparent quality factor produced by the damping and $Q_{e l}$ is the intrinsic quality factor of the electrical

matching network. The factor $\frac{Q_{a}}{Q_{e l}}$ in the thermal noise appears when the cold-damping network is active in the read-out circuit [1]. It comes of the fact that the damping is only a lossless electronic feedback effect and there is no dissipation associated to it. From Eqs. (43) we see that when the SQUID noise parameters $S_{v v}(T)$, and $S_{i i}(T)$ are known, and the impedance $Z(\omega)$ is measured, from the fit of the SQUID output current one can estimate as a fitting parameter the equivalent temperature of each transduction chain. One can measure 
the input impedance $Z(\omega)$ seen by the SQUID by injecting a sinewave signal with defined level through a calibration coil weakly coupled to the SQUID input circuit.

We can simulate numerically the calibration procedure. Denoting by $M_{c a l}$ the mutual inductance between the calibration coil and the SQUID input circuit and by $R_{\text {cal }}$ the resistance of the calibration line, to estimate the impedance $Z(\omega)$ one has to solve the system of equations in Eqs. (10). All the terms on the right hand side are zero but the SQUID input voltage, which is given by $V_{n}=\frac{j \omega M_{c a l}}{R_{c a l}} V_{c a l}$, with $V_{c a l}$ the voltage of the injected calibration signal. By measuring the output response of the SQUID amplifier we obtain a direct estimation of each transducer admittance from the following weighted average:

$$
\frac{1}{Z^{n n}(\omega)}=\frac{\operatorname{Re}\left\{I_{n} V_{n}^{*}\right\}+j \operatorname{Im}\left\{I_{n} V_{n}^{*}\right\}}{\left|V_{n}\right|^{2}}=G_{V_{n}}^{n n},
$$

where $I_{n}$ is the current at the input of each SQUID and $\mathrm{G}_{\mathbf{V}}^{\mathrm{nn}}$ was defined in Eqs. (21). The weighted average gives a more precise result than simply measuring $I_{n} / V_{n}$. We remark that both amplitude and phase of the input and output signal have to be measured to estimate $Z(\omega)$.

The admittance $1 / Z(\omega)$ can be approximated by a complex polynomial expansion with $N_{p}=5+2 \times N$ poles and $N_{q}=5+2 \times N-1$ zeros as follows

$$
\frac{1}{Z(\omega)}=A \frac{\prod_{k=1}^{N_{q}}\left(j \omega-q_{k}\right)\left(j \omega-q_{k}^{*}\right)}{\Pi_{k=1}^{N_{p}}\left(j \omega-p_{k}\right)\left(j \omega-p_{k}^{*}\right)}(j \omega)^{\left(N_{p}-N_{q}\right)} .
$$

If $\omega_{k}$ and $Q_{a, k}$ are the resonant frequency and the apparent Q-factor of each measured resonance oft he system, we have $p_{k}=j \omega_{k}-\omega_{k} / 2 Q_{a, k}$. The zeros $q_{k}$ can be written in the same form and have frequency and $\mathrm{Q}$ which depends on the modes coupling. Once the admittance is measured, a polynomial fit can be performed in order to find its zero and poles.

We simulated numerically the mode temperature calibration procedure. The current noise at the input of the SQUID amplifier was estimated from Eqs. (42) by calculating the impedance seen by the SQUID in the form of the admittance $1 / Z(\omega)$. For simplicity we only consider two resonators located in position 1 and position 2 of the TI arrangement. The detector is at $T=100 \mathrm{mK}$ with a $T / Q=2.5 \times 10^{-7}$, a SQUID energy resolution of $200 \hbar$ and an apparent quality factor $Q_{a}=600$. The electrical modes of each transducer are tuned to the mechanical modes. 


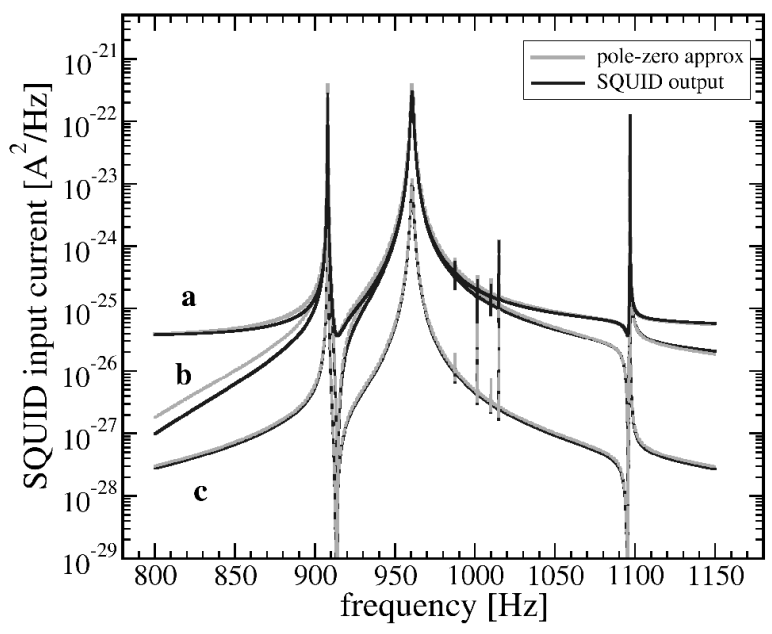

FIG. 20: Mode temperature calibration. The current noise density at the input of transducer 1 SQUID is estimated by calculation the admittance matrix $1 / Z(\omega)$. The contribution from thermal noise b), back-action noise c) and total noise including the additive SQUID current noise a) are shown. The dashed curves are obtained from the pole-zero polynomial expansion.

Figure 20 shows the SQUID input current noise contribution from the thermal and backaction noise and SQUID additive current noise of transducer line 1. The zero-pole approximation of Eqs. (45) is shown with dashed curves on top of the simulated current noise contributions.

\section{B. Force calibration and transfer functions measurements}

To calibrate a spherical detector one should be able to experimentally evaluate the transfer functions which relate the output current with the GW force acting on the spheroidal modes. When a force is applied at a point $\left(\theta_{c}, \phi_{c}\right)$ on the surface of the sphere, all the $5+2 N$ modes of the sphere and transducers are excited at an amplitude which depends on the calibrator position, the resonators positions and the coupling between resonators and spheroidal modes. A calibrator, consisting of a capacitive transducer with the main resonance frequency $\omega_{\text {cal }}$ not tuned with the fundamental frequencies $\omega_{0}$ of the detector, can be used to convert an electrical signal into a constant force acting on the sphere modes. For $\omega_{c a l}>>\omega_{0}$, such a force is given by

$$
F_{c}(\omega)=\frac{C_{c a l} E_{c a l}}{1-\frac{C_{c a l} E_{0}^{2}}{m_{c a l} \omega_{c a l}^{2}}} V_{c a l}(\omega),
$$


where $C_{c a l}, E_{c a l}$ and $m_{c a l}$ are respectively the capacitance, the dc bias electric field and the mass of the calibrator. $V_{c a l}(\omega)$ is the excitation voltage given to the calibrator at the resonance frequencies of the detector.

A force acting on the sphere surface always excites a combination of the 5 spheroidal modes. If denote by $\mathbf{F}_{\mathbf{c}}=\left(F_{1} \ldots F_{N c}\right)$ a vector of $N_{c}$ radial forces applied to the $N_{c}$ points $P_{c}=\left(\theta_{c}, \phi_{c}\right)$, with $c=1 \ldots N_{c}$, the corresponding forces $\mathbf{F}_{\mathbf{m}}$ acting on the 5 spheroidal modes can be described by the calibrators pattern matrix $\mathbf{B}_{\mathbf{C}}$ :

$$
\mathbf{F}_{\mathbf{m}}=\alpha \mathbf{B}_{\mathbf{C}} \mathbf{F}_{\mathbf{c}}
$$

From Eqs. (9) we find $B_{C m, c}=Y_{m, c}\left(\theta_{c}, \phi_{c}\right)$. The pattern matrix $\mathbf{B}_{\mathbf{C}}$ has the same physical meaning as the matrix B, but is referred to the calibrator positions. From Eqs. (47) it is clear that the gravitational forces acting on each of the five quadrupole modes, distributed over the entire volume, can be simulated by a linear combination of radial forces acting on the $N_{c} \geqslant 5$ calibrators. This conclusion is based on the assumption that the real spherical antenna dynamics is the same as that of an ideal sphere that can fully be modelled using the spherical harmonics approach described above. The validity of this assumption could be evaluated through a finite element analysis (FEA) of the detector structural model or by experimentally measuring the six transducers response to several linear combinations of excitation given through the calibrators.

To operate the sphere as a GW detector, only one force calibrator, mounted at an arbitrary position on the sphere surface, is necessary when $N=6$ transducers are used in the TI configuration. This is due to the existing one-to-one relation between the mode channels and the forces acting on the spheroidal modes. It is convenient to proceed by transforming the $N$ measured transducers outputs into the 5 mode channels, which directly describe the GW amplitude acting on the spheroidal modes [16]. In such a framework we derive the optimal filter for each mode channel to define an operative procedure to signal detection with a GW spherical resonant antenna. In order to do this we define the $5 \times 5$ mode channel noise spectral density matrix as

$$
\mathbf{S}_{\mathbf{I}, \mathbf{B}}(\omega)=\mathbf{B S}_{\mathbf{I}}(\omega) \mathbf{B}^{\dagger}
$$

and, using the admittance matrix $\mathbf{G}_{\mathbf{s i g}, \mathbf{I}}$ introduced in Eqs. (26), the $5 \times 1$ mode channel signal vector

$$
\mathbf{I}_{\mathrm{g}, \mathrm{sig}}=\mathbf{B I}_{\mathbf{i}}=\alpha \mathbf{B G}_{\mathbf{s i g}, \mathbf{I}} \mathbf{B}_{\mathbf{C}} \mathbf{F}_{\mathbf{c}}
$$


By applying a known constant force to the calibrator $\mathbf{F}_{\mathbf{c}}$, using Eqs. (477) we get the linear combination of spheroidal modes forces generated by the calibration force. By measuring simultaneously amplitude and phase of the six transducer outputs we obtain the mode channel response from Eqs. (49), and, using Eqs. (26), estimate the detector strain sensitivity. The matrix $\mathbf{S}_{\mathbf{I}, \mathbf{B}}(\omega)$ in Eqs. (48) is diagonal because the mode channels are statistically independent. The only assumption made here is that the the pattern matrix $\mathbf{B}$ derived above for an ideal sphere can be used to describe the dynamics of the real detector with six transducer in the TI arrangement. This can be experimentally verified in a separate cryogenic experiment, using for example a set of six calibrators in the TI configuration with a $60^{\circ}$ de-phase of the azimuthal angle with respect to the transducer arrangement. Each spheroidal mode force can be reproduced by simultaneously applying a linear combination of constant forces on the six calibrators, as described by Eqs. (47). When $N<5$ transducers are used we cannot transform the transducer outputs in mode channels. A set of at least 5 calibrators is then necessary to experimentally measure the transducer response to each mode channel. If only one calibrator is used, the detector output can only be calibrated for a particular combination of the five quadrupolar forces $F_{m}$.

Each element on the diagonal of $S_{I, B}^{(m)}=I_{m}(\omega) I_{m}^{*}(\omega)$ can be written in terms of polynomial ratio where the poles are the same as the ones derived in the impedance measurement of each transducers,see Eqs. (45), and the zeros depend on the current function $I_{m}(\omega)$. From the factorization of $I_{m}(\omega)$ one finds

$$
I_{m}(\omega)=S_{0, m}^{1 / 2} \Pi_{k=1}^{N_{p}} \frac{\left(j \omega-q_{k, m}\right)\left(j \omega-q_{k, m}^{*}\right)}{\left(j \omega-p_{k}\right)\left(j \omega-p_{k}^{*}\right)},
$$

where $S_{0, m}$ is the wideband noise of the $m^{\text {th }}$ mode channel, and equals the amplifier additive white noise if all the transducers SQUIDs are identical. From the definition in Eqs. (50), $S_{I, B}^{(m)}$ is real for real $\omega$ and the number of zeros and poles is the same as a consequence of assuming $S_{0}^{m}$ to be purely white.

The transfer functions for a GW signal, which convert the quadrupolar modes forces into mode channels currents according to Eqs. (49), contain the same poles $\left\{p_{k}\right\}$ and their factorization becomes

$$
H_{m}(\omega)=H_{m, c a l}(\omega) \frac{\prod_{k=1}^{N_{r}}\left(j \omega-r_{k, m}\right)\left(j \omega-r_{k, m}^{*}\right)}{\prod_{k=1}^{N_{p}}\left(j \omega-p_{k}\right)\left(j \omega-p_{k}^{*}\right)} .
$$

In the equation above $N_{p}>N_{r}$ and $H_{m, c a l}(\omega)$ is a force calibration constant which has to 
be experimentally determined at each cool down.

From now on we apply to each mode channel the standard Wiener-Kolmogorov (WK) filtering operation, developed so far for bar detectors [15]. We follow here the approach described in [23]. The best linear estimate of the amplitude $h_{0}$ of a given signal $h(t)$, with $\max$ $h(t)=h_{0}$ at the arrival time $t=0$, buried into an additive, zero mean, stationary gaussian noise $\eta$ can be obtained by correlating the mode channel output $Y_{m}(\omega)=\tilde{h}(\omega) H_{m}(\omega)+\eta(\omega)$ to the matched WK filter [39],

$$
W_{m}(\omega)=\sigma_{A}^{2} \frac{H_{m}^{*}(\omega) \tilde{h}^{*}(\omega)}{S_{I, B}^{(m)}(\omega)}
$$

where $\sigma_{A}^{2}=\int d \omega\left|H_{m}(\omega) \tilde{h}(\omega)\right|^{2} / S_{I, B}^{(m)}(\omega)$ is the variance of the noise after the filtering. The WK filter splits as the product $L_{m}(\omega) M_{m}(\omega) \tilde{h}^{*}(\omega)$, where $L_{m}(\omega)$ is a whitening filter for the noise $S_{I, B}^{(m)}(\omega)$ given by

$$
L_{m}(\omega)=I_{m}^{-1}(\omega)=S_{0, m}^{-1 / 2} \Pi_{k=1}^{N_{p}} \frac{\left(j \omega-p_{k, m}\right)\left(j \omega-p_{k, m}^{*}\right)}{\left(j \omega-q_{k, m}\right)\left(j \omega-q_{k, m}^{*}\right)} .
$$

$M_{m}$ is a bandpass filter around the frequencies $\omega_{k}=\left|\operatorname{Im}\left(q_{k, m}\right)\right|$ and bandwidths $\Delta \omega_{k}=$ $2 \operatorname{Re}\left(q_{k, m}\right)$. Such a bandwidth is generally much larger than the intrinsic bandwidth of each resonance and, in the case of a transducer with coupled electrical modes, it can reach values as large as $\sim 200 \mathrm{~Hz}$. One finds

$$
M_{m}(\omega)=\sigma_{A}^{2} \cdot S_{0, m}^{-1 / 2} \frac{\Pi_{k=1}^{N_{r}}\left(j \omega+r_{k, m}\right)\left(j \omega+r_{k, m}^{*}\right)}{\Pi_{k=1}^{N_{p}}\left(j \omega+q_{k, m}\right)\left(j \omega+q_{k, m}^{*}\right)} .
$$

The product $L_{m}(\omega) M_{m}(\omega)$ is the WK filter for a delta-like GW pulse. The extra term $\tilde{h}(\omega)$ should be added when a general GW signal $h(t)$ is considered. It can be shown that for a resonant bar detector, $N_{r}=1$ and $r_{1}=0$. In this case the WK filtering procedure is then fully defined by the zeros $q_{k}$ and poles $p_{k}$ of the noise power spectrum $S_{I}(\omega)$, the additive amplifier white noise $S_{0}$ and the calibration constant $H_{0}(\omega)$. For a spherical detector this is true only if the quadrupolar modes degenerate into a single resonant frequency and all the transducers have the same resonance. When multiple resonances are present, as is the case for real spherical detectors, the zeros in Eqs. (51) do not cancel i.e., $N_{r}>1$ and $r_{k, m} \neq 0$. This is due to the fact that when the quadrupolar modes are non-degenerate, a mixing occurs between mode channels. A fraction of the signal which should only go to one mode channel leaks into the others. The WK filtering procedure should include the extra set of 

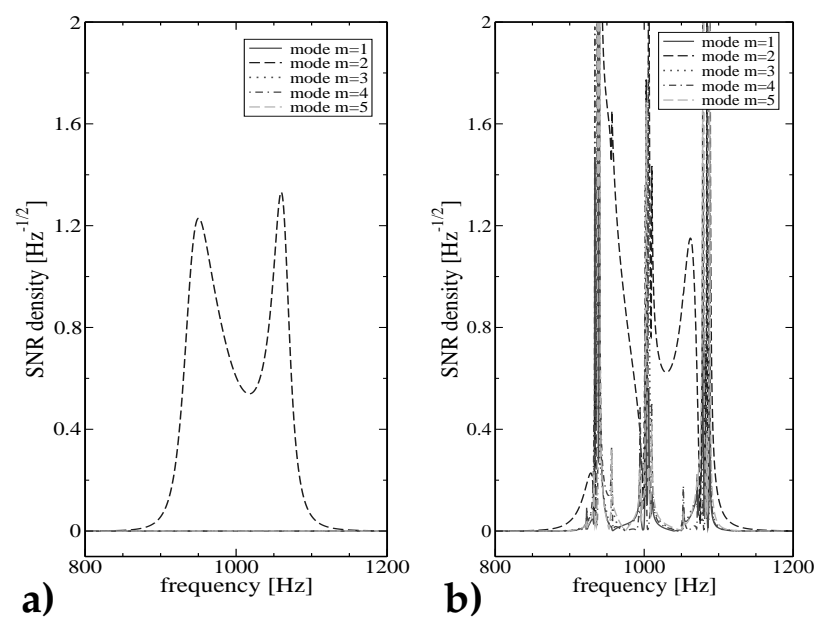

FIG. 21: Modes channels response from a simulated linearly polarized burst coming along the $z$ direction in the detector frame for a detector with degenerate, a), and non-degenerate, $\mathbf{b}$ ), modes. For a detector with degenerate modes, a), only the second mode gives a larger signal than the noise. For a real detector with non-degenerate modesb, a mixing between the modes is present around the resonances making the analysis more complex

parameters $r_{k, m}$, whose total number depends on the mode and transducer considered and must be experimentally determined.

In figure 21 the mode channels response is plotted for a linearly polarized burst coming along the detector $z$ direction. For a detector with degenerate modes, figure 21] a, only the second mode gives a larger signal than the noise, as can be expected for a signal from that particular direction and polarization. For a realistic detector with non-degenerate spheroidal modes a mixing between the modes is present around the resonances making the analysis more complex, see figure 21,b). However, the energy stored in the second mode, which can be derived from the integral of the SNR density, is larger than in the others and the incoming wave direction can still be reconstructed without any significant accuracy loss with respect to the degenerate case. To estimate the incoming wave direction we can use the approach derived in [12], using standard theory of signal detection. After being optimally filtered, the 5 mode channels generate a set of 5 amplitudes, $g_{m}$, for an input GW burst. The likelihood function for a detector with stationary and Gaussian noise is given by

$$
\lambda=\frac{1}{2 \pi} \prod_{m=1}^{5} \exp \left(-\frac{\left[h_{m}-g_{m}\right]^{2}}{\sigma_{m}^{2}}\right),
$$

where $h_{m}$ is the expected gravitational wave signal amplitude, $g_{m}$ is the mode channels 

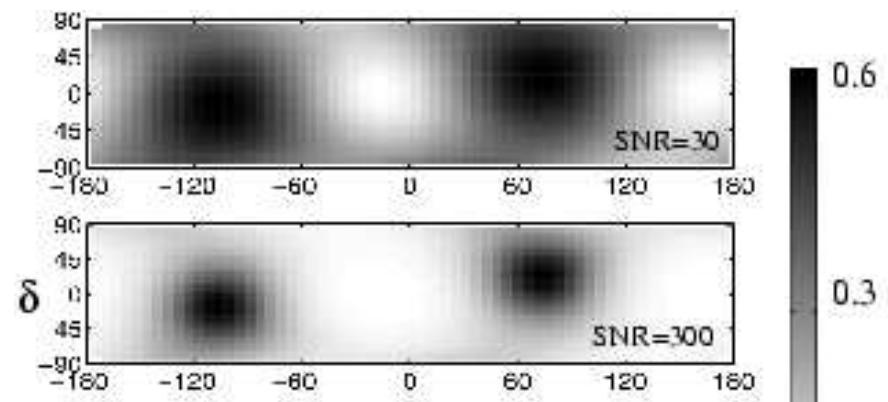

0.3

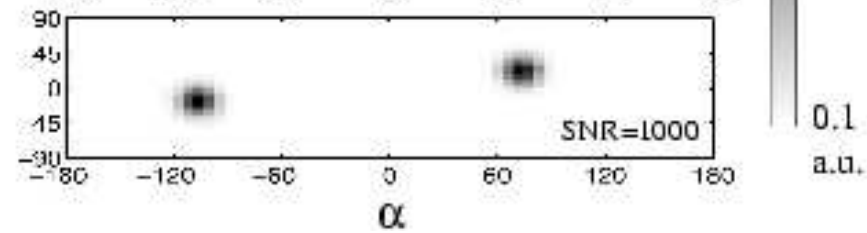

FIG. 22: Signal direction reconstruction. An overall sky search is performed calculating the likelihood function $\lambda$. We applied simulated burst signals with $S N R \sim 30,300,1000$, amplitude $h_{0}=0.34 \cdot 10^{-20}, 1.1 \cdot 10^{-20}, 2 \cdot 10^{-20}$ and $\tau_{G B} \sim 1 \mathrm{~ms}$, linearly polarized and coming from a source at declination $\delta=20^{\circ}$ and ascension $\alpha=70^{\circ}$. A single spherical detector cannot distinguish between sources laying in two opposite hemispheres.

amplitude obtained after the WK filtering procedure described above and $\sigma_{m}$ is the variance of $g_{m}$. A likelihood map is generated by plotting this function in the declination-ascension plane, $(\delta, \alpha)$. The maximum value of $\lambda$ gives to the estimated wave direction. In the example below, a realistic detector at the quantum limit, with optimized parameter is considered. We applied simulated burst signals, $1 \mathrm{~ms}$ long and with amplitude $h_{0}=0.34 \cdot 10^{-20}, 1.1$. $10^{-20}, 2 \cdot 10^{-20}$ and $S N R \sim 30,300,1000$, with linear polarization $h_{+}=h_{0}$ and $h_{\times}=0$, coming from a source at declination $\delta=20^{\circ}$ and ascension $\alpha=70^{\circ}$. Figure 22 plots the resulting likelihood functions.

The accuracy in the direction and polarization estimates depends on the SNR and is equal to $\Delta \Omega=2 \pi / S N R$ [12], 13]; it is direction independent when a sphere with more than 5 identical, point-like transducers is considered [44]. As it comes clear from the plot, a single spherical detector cannot distinguish between sources laying in the two opposite hemispheres. In the example described above we assumed for simplicity that the signal polarization were known. When this is not the case, one should include also the first Euler angle $\psi$ as a variable to estimate the likelihood ratio.

As discussed in [13], for a known signal, the optimal detection strategy for a vector output 
detector like a sphere with $\mathrm{N}$ transducers is to compute the value of the optimal linear filter with scalar output $\nu$ which maximizes the SNR. This procedure is useful if one wants to monitor the total energy in the sphere and claim a detection only on the base of an excess of absorbed energy. No direction information is possible in this way.

If we let $\mathbf{I}=I_{1} \ldots I_{N}$ be the transducers output stream, a linear filtering operation is performed which, in frequency domain, can be described by

$$
\nu(\omega)=\mathbf{W}^{\dagger}(\omega) \mathbf{I}(\omega),
$$

where $\mathbf{W}$ is a vector transfer function which maximizes the SNR and was found [13], to be

$$
\mathbf{W}(\omega)=\mathbf{I}_{\text {sig }}^{\dagger}(\omega) \mathbf{S}_{\mathbf{I}}^{-1}(\omega)
$$

In the above, $\mathbf{I}_{\mathbf{s i g}}=\mathbf{G}_{\mathbf{s i g}}(\omega) \mathbf{F}_{\mathbf{m}}^{\mathbf{S}}$ is the current generated in each transducer by a force $F_{m}^{S}$ on the sphere generated by a GW signal. As shown in [12], the maximum SNR for a multichannel detector is the sum of the maximum SNR of each individual channel. The optimal linear filter introduced above for each mode channel is then useful in order to compute the scalar output $\nu$ which maximizes the SNR.

\section{CONCLUSIONS}

We derived a complete and detailed electro-mechanical model for a spherical gravitational wave detector operating with multiple, two-mode capacitive transducers where the electrical resonant mode of a superconducting matching LC resonator can be tuned to the resonant modes. The signal current form the matching network is read out by SQUID amplifiers. The model allows to numerically calculate the sensitivity of a realistic detector and to study in detail the effects of the main mechanical and electrical parameters of the displacement read-out system on the strain sensitivity and bandwidth. All the known noise sources are discussed and considered in the model.

A complete numerical analysis has been performed for a 2 meter in diameter, 30 ton in mass, $\mathrm{CuAl}$ spherical detector operating at ultracryogenic temperatures. We derived the sensitivity for the spherical detector in its initial phase of development, when a single transducer is used, and when the detector operates with six transducers and becomes fully omnidirectional. The sensitivity is evaluated when the detector operates by making use of 
available technology and when it works at the quantum limit. We have shown that, in order to improve the strain sensitivity towards the quantum limit one should operate the detector at temperature of about $T=20 \mathrm{mK}$ with electrical and mechanical quality factor as high as $5 \cdot 10^{7}$ and massive mechanical resonators.

Direction anisotropies in the detector sensitivity and signal bandwidth are studied for a not-ideal detector operating with not identical, partially tuned and real-size resonators. The models made so far always consider rather generic, an point-like transducers, neglecting the fact that those sensors are in practice rather large and spatially distributed on a significant fraction of the sphere surface. We investigated here the validity of such an assumption.

Finally we described and numerically verify a complete calibration procedure, which makes use of techniques available for bar detectors. Similar algorithms can be used on spherical detector for diagnostic purpose and to derive the direction and polarization information from the detected signal.

\section{Acknowledgments}

The author would like to thank Massimo Bassan, Florian Dubath, Jean-Pierre Zendri and Alberto Lobo for reading this manuscript and for useful discussions. The MiniGRAIL project has been the major drive for writing this paper. This work has been financially supported by Integrated Large Infrastructures for Astroparticle Science (ILIAS) of the Sixth Framework Programme of the European Community.

[1] L. Baggio, M. Bignotto, M. Bonaldi, M. Cerdonio, L. Conti, P. Falferi, N. Liguori, A. Marin, R. Mezzena, A. Ortolan, et al., Phys. Rev. Lett. 94, 241101 (2005).

[2] P. Astone, R. Ballantini, D. Babusci, M. Bassan, P. Carelli, G. Cavallari, F. Cavanna, A. Chincarini, E. Coccia, C. Cosmelli, et al., Classical and Quantum Gravity 23, S57 (2006).

[3] A. de Waard, M. Bassan, Y. Benzaim, V. Fafone, J. Flokstra, G. Frossati, L. Gottardi, C. T. Herbschleb, A. Karbalai-Sadegh, K. Kuit, et al., Classical and Quantum Gravity 23, S79 (2006).

[4] O. D. Aguiar, L. A. Andrade, J. J. Barroso, F. Bortoli, L. A. Carneiro, P. J. Castro, C. A. Costa, K. M. F. Costa, J. C. N. de Araujo, A. U. de Lucena, et al., Classical and Quantum 
Gravity 23, S239 (2006).

[5] ROG collaboration, SFERA Project, unpublished (2005).

[6] M. Bonaldi, M. Cerdonio, L. Conti, M. Pinard, G. Prodi, and J. Zendri, Phys. Rev. D 68, 102004 (2003).

[7] M. Bonaldi, M. Cerdonio, L. Conti, P. Falferi, P. Leaci, S. Odorizzi, G. A. Prodi, M. Saraceni, E. Serra, and J. P. Zendri, Phys. Rev. D 74, 022003 (pages 13) (2006).

[8] N. Ashby and J. Dreitlein, Phys. Rev. D 12, 336 (1975).

[9] R. V. Wagoner and H. J. Paik, in Proceedings of the Pavia International Symposium, Pavia, Italy (Academia Nazionale dei Lincei, Rome,Italy, 1977).

[10] S. M. Merkowitz, Phys. Rev. D 58, 062002 (1998).

[11] J. Lobo, Phys. Rev. D 52, 591 (1995).

[12] C. Z. Zhou and P. F. Michelson, Phys. Rev. D 51, 2517 (1995).

[13] T. R. Stevenson, Phys. Rev. D 56, 564 (1997).

[14] J. C. Price, Phys. Rev. D 36, 3555 (1987).

[15] G. V. Pallottino and G. Pizzella, Il Nuovo Cimento 4C, 237 (1981).

[16] S. M. Merkowitz and W. W. Johnson, Phys. Rev. D 51, 2546 (1995).

[17] J. A. Lobo and M. A. Serrano, Europhys. Lett 35, 253 (1996).

[18] J. A. Lobo, Mon. Not. Roy. Astr. Soc. 316, 173 (2000).

[19] G. M. Harry, J. Houser, and K. A. Strain, Phys. Rev. D 65, 082001 (2002).

[20] P. Falferi, M. Bonaldi, M. Cerdonio, A. Vinante, R. Mezzena, and G. P. adn S. Vitale, Appl. Phys. Lett. 88, 062505 (2006).

[21] L. Gottardi, Ph.D. thesis, Leiden University, Leiden, The Netherlands (2004).

[22] A. Vinante, M. Bonaldi, P. Falferi, M. Cerdonio, R. Mezzena, G. Prodi, and S. Vitale, proceedings of the "SQUID 2001 Conference", Sept. 1-3, 2001, Stenungsbaden, Sweden, Physica C 368, 176 (2002).

[23] L. Baggio, M. Cerdonio, A. Ortolan, G. Vedovato, L. Taffarello, J. P. Zendri, M. Bonaldi, P. Falferi, V. Martinucci, R. Mezzena, et al., Phys. Rev. D 61, 102001 (2000).

[24] C. W. Misner, K. S. Thorne, and J. Wheeler, Gravitation (W. H. Freeman, San Francisco, 1973).

[25] P. Astone, D. Babusci, M. Bassan, P. Carelli, G. Cavallari, E. Coccia, C. Cosmelli, S. D’Antonio, V. Fafone, A. Fauth, et al., Phys. Rev. Lett. 91, 111101 (2003). 
[26] S. M. Merkowitz and W. W. Johnson, Phys. Rev. D 56, 7513 (1997).

[27] K. Yamamoto, S. Otsuka, Masaki, , K. Kawabe, and K. Tsubono, Class. Quantum Grav. 19, 1689 (2002).

[28] W. W. Johnson and S. M. Merkowitz, Phys. Rev. Lett. 70, 2367 (1993).

[29] C. W. Helstrom, Statistical Theory of Signal Detection (Pergamon, Oxford, 1968).

[30] F. Dubath, J. Extermann, and L. Gottardi, in preparation (2006).

[31] P. Saulson, Phys. Rev. D 42, 2437 (1990).

[32] E. Majorana and Y. Ogawa, Phys. Lett. A 233, 162 (1997).

[33] A. de Waard, Y. Benzaim, G. Frossati, L. Gottardi, H. van der Mark, J. Flokstra, M. Podt, M. Bassan, Y. Minenkov, A. Moleti, et al., Classical and Quantum Gravity 22, S215 (2005).

[34] Y. Levin, Phys. Rev. D 57, 659 (1998).

[35] T. Briant, M. Cerdonio, L. Conti, A. Heidmann, A. Lobo, and M. Pinard, Phys. Rev. D 67, 102005 (2003).

[36] P. Falferi, M. Cerdonio, L. Franceschini, R. Macchietto, S. Vitale, and J. P. Zendri, Rev. Sci. Instrum. 65, 2916 (1994).

[37] P. Falferi, M. Bonaldi, A. Cavalleri, M. Cerdonio, A. Vinante, R. Mezzena, K. Xu, G. A. Prodi, and S. Vitale, Appl. Phys. Lett. 82, 931 (2003).

[38] C. M. Caves, Phys. Rev. D 26, 1817 (1982).

[39] H. L. V. Trees, Detection, Estimation, and Modulation Theory. Part I (John Wiley and Sons, Inc, New York, 1968).

[40] N. Solomonson, W. W. Johnson, and W. O. Hamilton, Phys. Rev. D 46, 2299 (1992).

[41] M. Bassan, Y. Minenkov, and G. Zaccarian, in Proc. of the 1st Edoardo Amaldi Conf. on Gravitational Waves (World Scientific, Singapore, 1995), p. 358.

[42] A. de Waard, L. Gottardi, M. Bassan, E. Coccia, V. Fafone, J. Flokstra, A. Karbalai-Sadegh, Y. Minenkov, A. Moleti, G. V. Pallottino, et al., Classical and Quantum Gravity 21, S465 (2004).

[43] R. P. Giffard, Phys. Rev. D 14, 2478 (1976).

[44] S. M. Merkowitz, J. A. Lobo, and M. A. Serrano, Class. Quantum Grav. 16, 3035 (1999).

[45] N. Arnaud and M. Barsuglia, Phys. Rev. D 65, 0420041 (2004).

[46] To obtain large capacitance one could for example make use of a double electrode scheme as proposed in [41]. However, transducers with such a large mass and capacitance and high Q 
have never been developed so far and the feasibility needs to be demonstrated. 